\title{
An Exact Algorithm Based on MaxSAT Reasoning for the Maximum Weight Clique Problem
}

\author{
Zhiwen Fang \\ State Key Lab. of Software Development Environment \\ Beihang University, Beijing, 100083, P.R. China
}

Chu-Min Li

CHU-MIN.LI@U-PICARDIE.FR

MIS, Université de Picardie Jules Verne

Amiens 80039, France

Ke Xu

KEXU@NLSDE.BUAA.EDU.CN

State Key Lab. of Software Development Environment

Beihang University, Beijing, 100083, P.R. China

\begin{abstract}
Recently, MaxSAT reasoning is shown very effective in computing a tight upper bound for a Maximum Clique (MC) of a (unweighted) graph. In this paper, we apply MaxSAT reasoning to compute a tight upper bound for a Maximum Weight Clique (MWC) of a wighted graph. We first study three usual encodings of MWC into weighted partial MaxSAT dealing with hard clauses, which must be satisfied in all solutions, and soft clauses, which are weighted and can be falsified. The drawbacks of these encodings motivate us to propose an encoding of MWC into a special weighted partial MaxSAT formalism, called LW (Literal-Weighted) encoding and dedicated for upper bounding an MWC, in which both soft clauses and literals in soft clauses are weighted. An optimal solution of the LW MaxSAT instance gives an upper bound for an MWC, instead of an optimal solution for MWC. We then introduce two notions called the Top- $k$ literal failed clause and the Top- $k$ empty clause to extend classical MaxSAT reasoning techniques, as well as two sound transformation rules to transform an LW MaxSAT instance. Successive transformations of an LW MaxSAT instance driven by MaxSAT reasoning give a tight upper bound for the encoded MWC. The approach is implemented in a branch-and-bound algorithm called MWCLQ. Experimental evaluations on the broadly used DIMACS benchmark, BHOSLIB benchmark, random graphs and the benchmark from the winner determination problem show that our approach allows MWCLQ to reduce the search space significantly and to solve MWC instances effectively. Consequently, MWCLQ outperforms state-of-the-art exact algorithms on the vast majority of instances. Moreover, it is surprisingly effective in solving hard and dense instances.
\end{abstract}

\section{Introduction}

Consider an undirected graph $G=(V, E)$, where $V$ is a set of $n$ vertices $\left\{v_{1}, v_{2}, \ldots, v_{n}\right\}$ and $E$ is a set of $m$ edges. The density of $G$ is computed as $2 m /(n(n-1))$. A clique of $G$ is a subset $C \subseteq V$ in which every pair of vertices is adjacent. On the contrary, an independent set of $G$ is a subset $I \subseteq V$ in which every pair of vertices is disconnected. A vertex cover of $G$ is a subset $S \subseteq V$ such that every edge in $G$ has at least one endpoint in $S$. The maximum clique (MC) problem asks to find a clique with the largest cardinality. The MC problem is a prominent combinatorial optimization problem and it is tightly related to two other well-known graph problems, namely the maximum 
independent set (MIS) problem and the minimum vertex cover (MVC) problem. Concretely, a maximum clique $C$ of $G$ is a maximum independent set of the complement graph $\bar{G}$ of $G$, and $V \backslash C$ is a minimum vertex cover of $\bar{G}$. Therefore, algorithms for any of the three problems can directly be applied to solve the others in practice. In addition, most exact MC solvers take advantage of the following relation between the size of a maximum clique and the number of independent sets. If $G$ can be partitioned into $k$ independent sets, then $G$ cannot contain a clique larger than $k$, because an independent set can contribute at most one vertex to a clique.

The MC problem is NP-hard and its decision problem is NP-complete (Karp, 1972), which appears in many applications such as social network analysis (e.g., Zhang, Nie, Jiang, Chen, \& Liu, 2014b; Kibanov, Atzmueller, Scholz, \& Stumme, 2014). It is fixed-parameter intractable (Downey $\&$ Fellows, 1995). Moreover, it is proved that approximating MC within $|V|^{1-\varepsilon}$ for any given $\varepsilon>0$ is NP-hard (Zuckerman, 2006). The best polynomial-time approximation algorithm achieves an approximation ratio of $O\left(n(\log \log n)^{2} /(\log n)^{3}\right)$ (Feige, 2004). Because of the theoretical and practical importance of the MC problem, a huge amount of effort has been devoted to solve it by designing two types of algorithms (also called solvers). One type is heuristic algorithms mainly including stochastic local search (e.g., Pullan \& Hoos, 2006; Cai, Su, \& Sattar, 2011; Cai, Su, Luo, \& Sattar, 2013; Fang, Chu, Qiao, Feng, \& Xu, 2014a). Another is exact algorithms including branch-and-bound (BnB) search (e.g., Östergård, 2002; Régin, 2003; Tomita \& Seki, 2003; Konc \& Janezic, 2007; Li \& Quan, 2010b; Tomita \& Kameda, 2007; Li, Fang, \& Xu, 2013). Heuristic algorithms are able to solve large-scale instances but cannot guarantee the optimality of their solutions. Exact algorithms guarantee the optimality of their solutions, but the worst-case time complexity is exponential unless $P=N P$.

A tight upper bound of the size of a maximum clique in a graph is essential for a $\mathrm{BnB}$ algorithm to solve the MC problem efficiently. However, it is very challenging to obtain such an upper bound in reasonable time. Most state-of-the-art BnB algorithms apply approximation coloring and independent set partition algorithms to compute an upper bound for MC. For instance, Fahle (2002) uses the constructive heuristic DSATUR to color vertices one by one according to their degrees. Konc and Janezic (2007), Tomita and Kameda (2007), Li and Quan (2010b) apply the greedy strategy proposed by Tomita and Seki (2003) to partition the graph into independent sets, and use the number of independent sets in the partition as an upper bound for MC. MaxCLQ (Li \& Quan, 2010b, 2010a) encodes an MC instance into a partial MaxSAT instance and improves the upper bound based on the independent set partition by making use of MaxSAT reasoning. The excellent performance of MaxCLQ shows that MaxSAT reasoning technologies allows to compute a tight upper bound for MC within reasonable time. IncMaxCLQ (Li et al., 2013) combines an incremental upper bound and MaxSAT reasoning to compute a tight upper bound more efficiently. In addition to the independent set partition and MaxSAT reasoning, other approaches, such as the graph matching (Régin, 2003), are also used in the upper bounding for MC.

One generalization of the MC problem is to associate each vertex with a positive weight. The weight of a clique is defined as the total weight of vertices in it. The maximum weight clique (MWC) problem consists in finding a clique with the largest weight. It is computationally equivalent to problems like the weighted set packing problem. The MWC problem appears in a variety of realworld applications, such as protein structure predictions (Mascia, Cilia, Brunato, \& Passerini, 2010), coding theory (Zhian, Sabaei, Javan, \& Tavallaie, 2013), combinatorial auctions (Wu \& Hao, 2015), computer vision (Ma \& Latecki, 2012; Zhang, Javed, \& Shah, 2014a), etc. For example, in the video object segmentation problem, a challenging task is to select a region having high objectness 
score and sharing similar appearance, which can be solved by an MWC algorithm (Ma \& Latecki, 2012).

Compared to the MC problem, less work has been done to solve the MWC problem. Cliquer (Östergård, 2002, 2001) is one of the few state-of-the-art exact solvers for both MC and MWC, and it deals with MC and MWC using similar methods. In a preprocessing, Cliquer partitions the graph into independent sets by determining one independent set at a time. As long as there are vertices that can be added into the current independent set, the one with the largest degree is added. The purpose of this preprocessing is to define a vertex ordering $v_{1}<v_{2}<\ldots<v_{n}$, where $v_{i}(1 \leq i \leq n)$ is the vertex inserted into an independent set at time $i$. Then Cliquer searches for an MC or MWC in the subgraph induced by $\left\{v_{i}, v_{i+1}, \ldots, v_{n}\right\}$ successively for $i=n, n-1, \ldots, 1$ (in this ordering). The MC or the MWC in the subgraph induced by $\left\{v_{i}, v_{i+1}, \ldots, v_{n}\right\}$ is associated with $v_{i}$ and is used to prune subtrees in subsequent search. Kumlander $(2004,2008 \mathrm{~b})$ inherits the search strategy from Cliquer. In addition to the MWC associated with $v_{i}$, Kumlander also partitions the current subgraph into independent sets and uses the sum of the maximum weight in each independent set as an upper bound. VCTable (Shimizu, Yamaguchi, Saitoh, \& Masuda, 2012) improves Kumlander's algorithm by a new initial vertex order and a better implementation using bitwise operations. Yamaguchi and Masuda (2008) propose a new upper bound based on the longest path in a directed acyclic graph constructed from the original graph, which improves the bound based on the independent set partition. OTClique (Shimizu, Yamaguchi, Saitoh, \& Masuda, 2013), which is also based on Cliquer, uses a dynamic programming strategy to calculate upper bounds of some small subproblems in a preprocessing, and stores all the results in a table. The stored upper bounds are used during search. MinSatz (Li, Zhu, Manyà, \& Simon, 2012) is an exact solver for the MinSAT problem. An important application of MinSatz is to solve combinatorial optimization problems such as MC and MWC. MinSatz constructs a weighted graph for the MinSAT instance and uses the clique partition combined with MaxSAT reasoning to compute a tight bound for the MinSAT instance to solve. In addition to exact algorithms, some heuristic algorithms are also proposed to solve the MWC problem (Pullan, 2008; Wu, Hao, \& Glover, 2012; Benlic \& Hao, 2013).

In this paper, we apply MaxSAT reasoning to solve MWC. We first study three usual encodings of MWC into MaxSAT. All these encodings have intrinsic difficulties in dealing with vertex weights. This motivates us to propose a dedicated encoding of MWC into MaxSAT called $L W$ (Literal-Weighted) encoding, in which both soft clauses and literals in soft clauses are weighted. While an optimal solution of a MaxSAT instance by usual encodings of MWC into MaxSAT gives an MWC, an optimal solution of an LW MaxSAT instance by the LW encoding just gives an upper bound for the MWC. So, it makes little sense to run a MaxSAT solver to find the optimal solution of a LW MaxSAT instance. The interest of the LW encoding is that we can transform an LW MaxSAT instance to reduce its optimal solution, so that a tighter upper bound for the encoded MWC can be obtained.

In order to transform an LW MaxSAT instance, we introduce two notions called the Top-k literal failed clause and the Top-k empty clause, and two sound transformation rules. Then, we implement a BnB algorithm for MWC called MWCLQ. In every search tree node, MWCLQ first encodes the current subgraph into an LW MaxSAT instance. Then, driven by MaxSAT reasoning, MWCLQ repeatedly transforms the LW MaxSAT instance to obtain a tighter upper bound for the encoded MWC. To the best of our knowledge, it is the first time that MaxSAT reasoning techniques are used specifically to compute a tight upper bound in a BnB algorithm for MWC. Experimental results on the widely used DIMACS benchmark, BHOSLIB benchmark, random graphs and realistic 
benchmark from the winner determination problem show that MWCLQ reduces the search space significantly and outperforms the state-of-the-art exact algorithms on the vast majority of instances from those benchmarks.

This paper is extended from the work of Fang, Li, Qiao, Feng, and Xu (2014b) by:

- clearly motivating the LW encoding by illustrating the weakness of three classical encodings of MWC into MaxSAT in dealing with vertex weights of a graph;

- introducing the notion Top- $k$ empty clause and exploiting it in MWCLQ;

- formally proving the transformation rules;

- adding more experimental results to show the effectiveness of our approach. The algorithm MWCLQ is now compared with the integer programming solver CPLEX, and the MinSAT solver MinSatz, especially on realistic instances from the winner determination problem. State-of-the-art MaxSAT solvers using different encodings of MWC into MaxSAT are also compared with MWCLQ. In order to further evaluate the LW encoding, which the state-ofthe-art MaxSAT solvers cannot use, we compare different versions of MWCLQ, in which the only difference is the encoding of MWC into MaxSAT used to compute the upper bound.

This paper is organized as follows. In the next section, we introduce some necessary notations and background knowledge. In Section 3, we present MWCLQ and different encodings of MWC into MaxSAT, before extending MaxSAT reasoning to weighted literals by introducing the notions of Top- $k$ literal failed clause and Top- $k$ empty clause, and two transformation rules. Experimental results are shown in Section 4. Section 5 concludes the paper.

\section{Preliminaries}

The subgraph of $G$ induced by a subset $V^{\prime} \subseteq V$ is $G^{\prime}=\left(V^{\prime}, E^{\prime}\right)$, where $E^{\prime}=\left\{\left\{v_{i}, v_{j}\right\} \mid v_{i}, v_{j} \in V^{\prime} \wedge\right.$ $\left.\left\{v_{i}, v_{j}\right\} \in E\right\}$. For a vertex $v, \Gamma(v)=\{u \mid\{u, v\} \in E\}$ is the set of neighbors of $v$ and the cardinality $|\Gamma(v)|$ is called the degree of $v$. We use $G_{v}$ to denote the subgraph induced by $\Gamma(v) \cup\{v\}$ and $G \backslash v$ to denote the subgraph induced by $V \backslash\{v\}$. A maximal clique is a clique that cannot be extended any more. A maximum clique is a maximal clique of the largest possible size. The cardinality of a maximum clique of $G$ is usually denoted as $\omega(G)$ and is called the clique number of $G$. For any vertex $v$ in $G$, a maximum clique of $G$ can be either in $G_{v}$ or in $G \backslash v$. The chromatic number of $G$, denoted by $\chi(G)$, is the minimum number of colors needed to color the vertices of $\mathrm{G}$ such that no two adjacent vertices share the same color. The vertices sharing the same color constitute an independent set. Therefore, the graph coloring problem is equivalent to partitioning $V$ into a minimum number of independent sets. Note that $\chi(G)$ is greater than or equal to $\omega(G)$.

A graph can be edge-weighted or vertex-weighted. We focus on the vertex-weighted graph in this paper. Formally, a vertex-weighted undirected graph $G=(V, E, w)$ is an undirected graph $G=$ $(V, E)$ combined with a weighting function $w: V \rightarrow R^{+}$such that every vertex $v$ is associated with a positive weight $w(v)$. In the sequel, we use the term weighted graph instead of vertex-weighted graph for simplicity. The weight of a clique $C$ in $G$ is defined to be $w(C)=\sum_{v \in C} w(v)$. Given a weighted graph $G$, the maximum weight clique problem asks to find a clique with the largest weight in $G$ (Östergård, 2001; Pullan, 2008; Wu et al., 2012) and this largest weight is often denoted by 
$\omega_{v}(G)$ in the literature. Note that a maximum weight clique is not necessarily a clique containing the maximum number of vertices, but it must be a maximal clique.

The MC problem can be encoded into a partial MaxSAT problem. In MaxSAT, a variable $x$ may take value 0 ( false) or 1 (true). A literal $\ell$ is a variable $x$ or its negation $\bar{x}$. A clause $c=\ell_{1} \vee \ell_{2} \vee$ $\ldots \vee \ell_{l}$ is a disjunction of literals, which can also be expressed as a set $\left\{\ell_{1}, \ell_{2}, \ldots, \ell_{l}\right\}$. A clause is satisfied if and only if the clause has at least one literal assigned to true. The length of clause $c$ is the number of literals it contains, denoted by length $(c)$. A unit clause is a clause containing only one literal. An empty clause, denoted by $\square$, contains no literals and cannot be satisfied. A conjunctive normal form (CNF) formula $\phi=c_{1} \wedge c_{2} \wedge \ldots \wedge c_{m}$ is a conjunction of clauses. Given a CNF formula $\phi$ on the set of variables $\left\{x_{1}, x_{2}, \ldots, x_{n}\right\}$, the satisfiability (SAT) problem is to test if there is an assignment satisfying all the clauses of $\phi$, and the maximum satisfiability (MaxSAT) problem is to find an assignment satisfying the maximum number of clauses (Li \& Manya, 2009). The minimum satisfiability (MinSAT) problem, on the contrary, is to find an assignment minimizing the number of satisfied clauses ( $\mathrm{Li}$ et al., 2012). In some cases, some clauses can be declared to be hard and must be satisfied in all solutions, and other clauses are soft and can be falsified. The partial MaxSAT problem asks to find an assignment to maximize the number of satisfied soft clauses while satisfying all hard clauses. Note that the MaxSAT problem is a particular partial MaxSAT problem without hard clauses. A weighted clause is a pair $(c, w)$, where $c$ is a soft clause and $w$, a positive number, is its weight. A weighted partial MaxSAT problem is to find an assignment maximizing the total weight of satisfied soft clauses while satisfying all hard clauses. A weighted MaxSAT problem is a weighted partial MaxSAT problem without hard clauses. The optimal solution of a (weighted) (partial) MaxSAT instance $\phi$ is denoted as $\operatorname{opt}(\phi)$ in this paper.

Two main types of exact algorithms are developed for MaxSAT: SAT-based MaxSAT solvers (e.g., Morgado, Heras, Liffiton, Planes, \& Marques-Silva, 2013; Ansótegui, Bonet, \& Levy, 2013; Davies \& Bacchus, 2013a; Ansótegui \& Gabas, 2013; Morgado, Dodaro, \& Marques-Silva, 2014; Martins, Joshi, Manquinho, \& Lynce, 2014) that solve a MaxSAT instance by repeatedly calling a CDCL (Conflict-Driven Clause Learning) based SAT solver to solve a sequence of SAT problems, and the BnB MaxSAT solvers (e.g., Li, Manyà, \& Planes, 2007; Kügel, 2010). The SAT-based MaxSAT solvers are particularly efficient to solve industrial MaxSAT problems, while the BnB MaxSAT solvers are particular efficient to solve the random MaxSAT problems. Some SAT-based solvers such as MaxHS (Davies \& Bacchus, 2013b) also exploit MIP (Mixed Integer Programming) for solving MaxSAT.

To maximize the number of satisfied clauses equals to minimize the number of falsified clauses. Many algorithms based on the BnB scheme for MaxSAT compute a lower bound of the number of falsified clauses (Li, Manya, \& Planes, 2006; Li et al., 2007; Larrosa, Heras, \& de Givry, 2008; Kügel, 2010) to prune the search space when solving a MaxSAT instance $\phi$. Detecting disjoint inconsistent subsets of soft clauses is proved to be very powerful in computing such a bound, where a subset of soft clauses is said inconsistent if this subset together with hard clauses is unsatisfiable. Unit propagation is an effective technique widely used in SAT and MaxSAT solvers (Li \& Anbulagan, 1997; Li, Manyà, \& Planes, 2005). The pseudo-code allowing to find an inconsistent subset of soft clauses based on unit propagation is given in Algorithm 1 ( $\mathrm{Li}$ et al., 2005). The algorithm works as follows. It uses a stack $S$ to store all unit clauses of $\phi$ and performs unit propagation until an empty clause is produced or $S$ is empty. If an empty clause is produced, the set of all clauses used in deriving the empty clause, excluding the hard clauses, is returned. The algorithm is called iteratively to find as many disjoint inconsistent subsets of soft clauses as possible. Note that soft 
clauses involved in an inconsistent subset are removed before detecting other inconsistent subsets to ensure that these subsets are disjoint. Also note that $\phi$ does not have any solution if an empty set is returned, because the empty set means that the set of hard clauses is unsatisfiable.

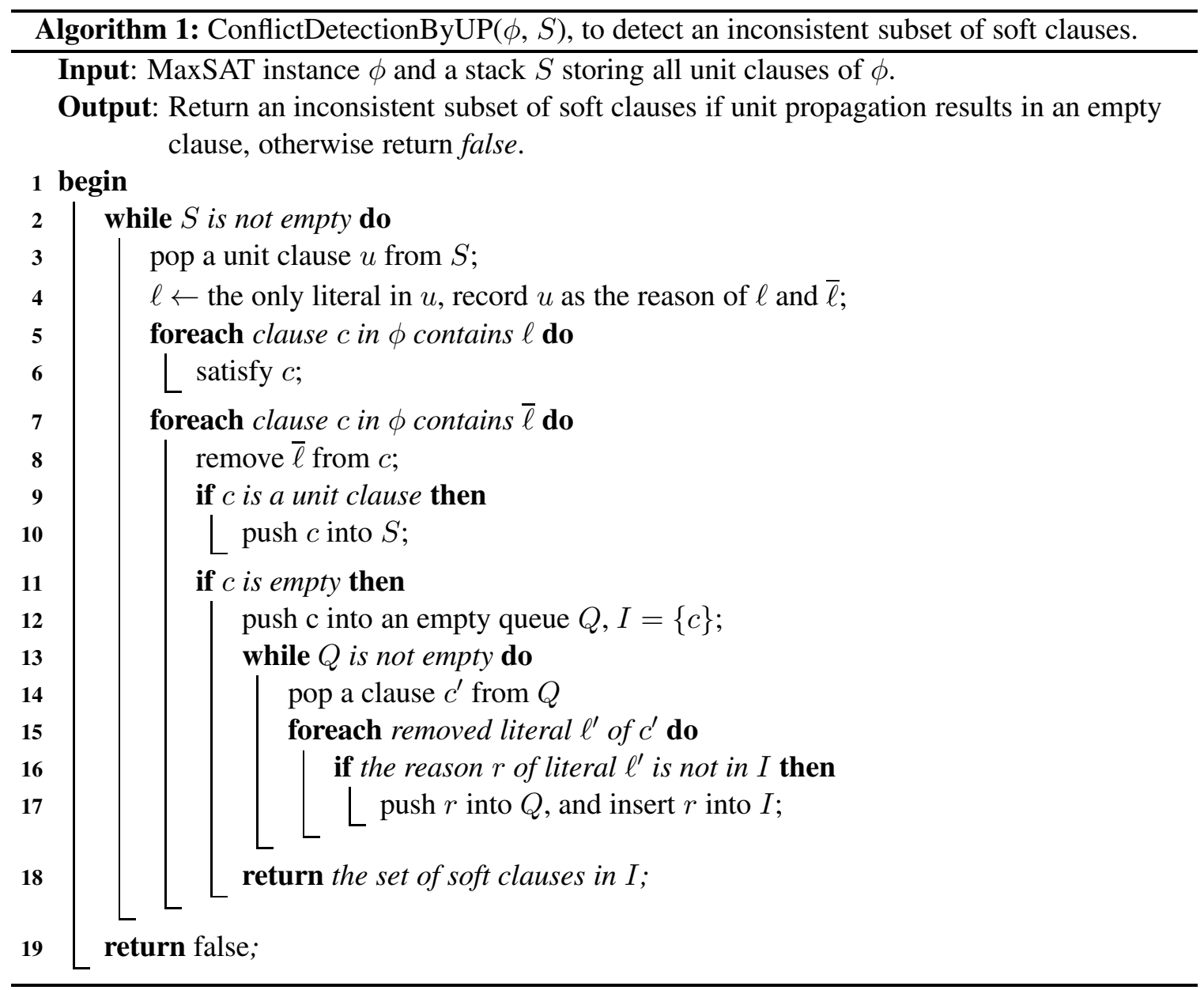

Failed literal detection (Freeman, 1995) is used to enhance unit propagation in MaxSAT solving ( $\mathrm{Li}$ et al., 2006). A literal $\ell$ of a CNF formula $\phi$ is called a failed literal of $\phi$, if unit propagation in $\phi \cup\{\ell\}$ results in an empty clause. Let $I S(\ell)$ be the set of soft clauses used in the unit propagation to derive an empty clause after assigning true to $\ell$. If all literals in a soft clause $c=\left\{\ell_{1}, \ell_{2}, \ldots, \ell_{l}\right\}$ are failed, then $\{c\} \cup I S\left(\ell_{1}\right) \cup I S\left(\ell_{2}\right) \cup \cdots \cup I S\left(\ell_{l}\right)$ is an inconsistent subset of soft clauses ( $\mathrm{Li} \&$ Quan, 2010b).

\section{MWCLQ: An Exact Algorithm for MWC}

In this section, we propose an exact algorithm based on the BnB scheme, namely MWCLQ, for MWC. In Subsection 3.1, we describe a basic BnB algorithm. In Subsection 3.2, we introduce a novel encoding called LW (Literal-Weighted) encoding of MWC into MaxSAT after discussing three usual encodings. Given a weighted graph $G$, the LW MaxSAT encoding gives an LW MaxSAT instance $\phi_{l w}$ that represents an upper bound of $\omega_{v}(G)$. In Subsection 3.3, we propose two transfor- 
mation rules for $\phi_{l w}$. Successive transformations of $\phi_{l w}$ driven by MaxSAT reasoning give a tight upper bound of $\omega_{v}(G)$, which is presented in Subsection 3.4.

\subsection{Branch-and-Bound Search for MWC}

Algorithm 2 depicts the pseudocode of MWCLQ.

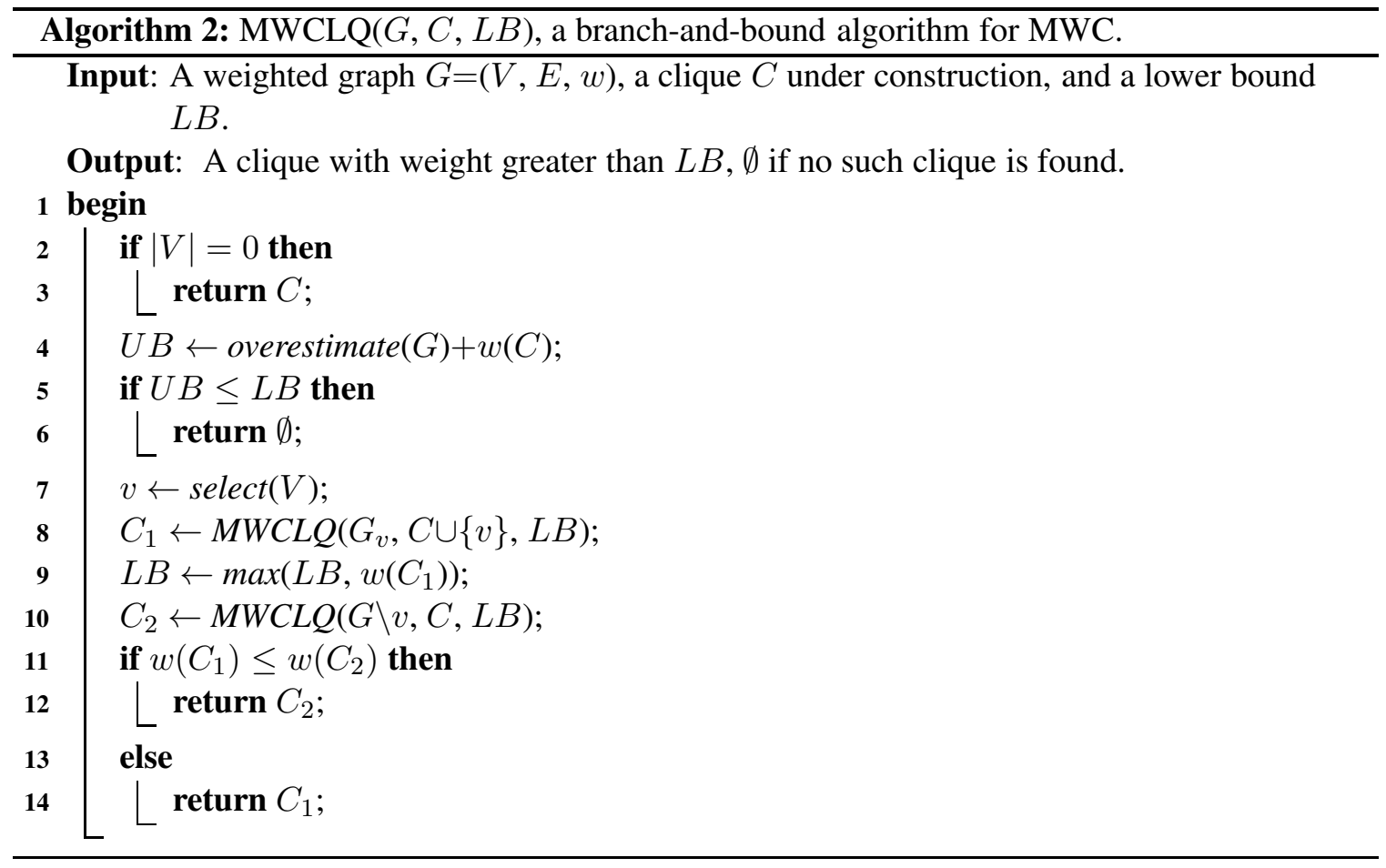

MWCLQ searches for a clique, extended from $C$, with weight larger than $L B$ in $G=(V, E$, $w)$. It first computes an upper bound $U B$ by calling overestimate $(G)$ and then compares $U B$ with $L B$ to test whether further search is necessary in $G$. If it is possible to find a better solution, MWCLQ selects a vertex by calling $\operatorname{select}(V)$. For any vertex $v$ in $G$, a maximum weight clique of $G$ is either a clique in $G_{v}$ containing $v$ or a clique in $G \backslash v$ not containing $v$. Thus, MWCLQ searches for a maximum weight clique in $G_{v}$ and in $G \backslash v$ successively.

MWCLQ has a form similar to MaxCLQ, a BnB algorithm for MC (Li \& Quan, 2010b), but the overestimate function for computing an upper bound, and the select function for choosing a branching vertex, are significantly different. These two functions are essential for both MWCLQ and MaxCLQ. A high-quality upper bound allows solvers to prune useless search, and a good vertex ordering promises an efficient search process. MaxCLQ computes a base upper bound by partitioning $G$ into independent sets, and then improves the base upper bound by MaxSAT reasoning. Meanwhile, MaxCLQ orders vertices by selecting first the vertex with minimum degree. In this paper, we use a simple vertex ordering to select $v$ with the largest weight, breaking ties in favor of the vertex with higher degree, and focus on how to efficiently compute a tight upper bound using MaxSAT reasoning. 


\subsection{Encoding MWC into MaxSAT}

An MC or MWC instance can be encoded into MaxSAT as follows.

Boolean variables: A boolean variable $x_{i}$ is added for each vertex $v_{i}$, which is assigned the value true if and only if $v_{i}$ is in the maximum clique under construction;

Hard clauses: A set of hard clauses is added to require that any pair of unconnected vertices does not belong to the same clique. Concretely, a hard clause $\bar{x}_{i} \vee \bar{x}_{j}$ is added for each pair of unconnected vertices $v_{i}$ and $v_{j}$;

Soft clauses: There exist different ways to define the set of soft clauses. Given a graph, all encodings of MC or MWC into MaxSAT presented in this paper use the same set of hard clauses. They differ only in the soft clauses used, and will be analyzed and discussed in this subsection.

An MC instance can also be encoded into a MinSAT instance without hard clauses by adopting the approach proposed by Ignatiev, Morgado, and Marques-Silva (2014). The obtained MinSAT instance can be in turn encoded into a MaxSAT instance without hard clauses using the approaches from the work of Kügel (2012), Zhu, Li, Manyà and, Argelich (2012). This encoding without hard clauses provides a new angle of view for the MC or MWC solving, which awaits future research. In this section, we focus on encodings of MWC into MaxSAT with the hard clauses and only different in the soft clauses used. Subsection 3.2.1 defines the direct encoding of MWC into MaxSAT. Subsection 3.2.2 defines split encoding and iterative split encoding of MWC for MaxSAT. Subsection 3.2.3 proposes the novel literal-weighted encoding of MWC into MaxSAT and illustrates its advantages compared with the direct encoding, the split encoding and the iterative split encoding in computing the upper bound for MWC.

\subsubsection{Direct EnCODING OF MWC InTO MAXSAT}

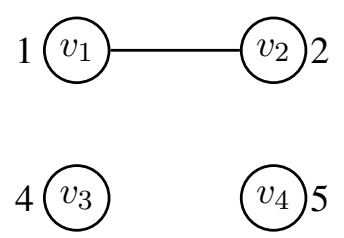

Figure 1: A weighted graph with 4 vertices and 1 edge. Numbers indicate vertex weights.

A straightforward way to define soft clauses is to associate a unit soft clause $x_{i}$ with each vertex $v_{i}$, giving the direct encoding of MC or MWC into MaxSAT. For example, the MC instance for the graph in Fig. 1 (without considering vertex weights) is encoded into the following partial MaxSAT instance: (1) the set of variables is $\left\{x_{1}, x_{2}, x_{3}, x_{4}\right\}$; (2) the set of hard clauses is $\left\{\bar{x}_{1} \vee \bar{x}_{3}, \bar{x}_{1} \vee \bar{x}_{4}\right.$, $\left.\bar{x}_{2} \vee \bar{x}_{3}, \bar{x}_{2} \vee \bar{x}_{4}, \bar{x}_{3} \vee \bar{x}_{4}\right\}$; and (3) the set of soft clauses is $\left\{x_{1}, x_{2}, x_{3}, x_{4}\right\}$.

Any assignment satisfying all hard clauses gives rise to a clique, since the variables assigned the value true correspond to pairwise connected vertices. For the non-weighted case, an assignment satisfying all hard clauses and maximizing the number of satisfied soft clauses gives rise to a maximum clique. 
For the weighted case, each unit soft clause is associated with the weight of the corresponding vertex. For example, the MWC instance for the weighted graph in Fig. 1 can be encoded into a weighted partial MaxSAT instance with the same boolean variables and the same hard clauses as in the MC instance. The set of weighted soft clauses is: $\left\{\left(x_{1}, 1\right),\left(x_{2}, 2\right),\left(x_{3}, 4\right),\left(x_{4}, 5\right)\right\}$. An assignment satisfying all hard clauses and maximizing the total weight of satisfied soft clauses gives rise to a maximum weight clique.

Once encoded into MaxSAT, MaxSAT reasoning can be applied to solve the MC or MWC problem. Many MaxSAT algorithms obtain an upper bound of satisfied soft clauses by computing a lower bound of the number of falsified soft clauses. Inconsistent subsets of soft clauses are often used for computing such a lower bound. Recall that a subset of soft clauses is said inconsistent if the subset together with hard clauses is not satisfiable. For a non-weighted MaxSAT instance $\phi$ with $m$ soft clauses, if $r$ disjoint inconsistent subsets of soft clauses are detected, then $m-r$ is an upper bound of $\operatorname{opt}(\phi)$ (Li et al., 2005, 2006).

For the weighted case, we define the weight of a subset $S$ of soft clauses to be the minimum weight of clauses in $S$, namely,

$$
w(S)=\min _{c \in S} w(c)
$$

Similar to the non-weighted case, we have the following proposition.

Proposition 1. (Li et al., 2007) Given a weighted partial MaxSAT instance $\phi$, if s disjoint inconsistent subsets $S_{1}, S_{1}, \ldots, S_{s}$ are detected, then opt $(\phi) \leq \sum_{\text {soft clause } c \in \phi} w(c)-\sum_{1 \leq i \leq s} w\left(S_{i}\right)$.

Observe that unit soft clauses of the direct encoding do not capture any connection between vertices, because the same set of soft clauses is used for every graph with $n$ vertices, no matter how these vertices are connected between each other. For an MC instance, the number of soft clauses is used as a base upper bound, and then MaxSAT reasoning is applied to improve the base upper bound by detecting inconsistent soft clause subsets. Unfortunately, the direct encoding can only give a trivial base upper bound, which is the number of vertices in the graph. Moreover, since all soft clauses of the direct encoding are unit, an inconsistent subset contains exactly two soft clauses, limiting the improvement of the upper bound to the half of the number of vertices in the graph. The weighted case is similar. The base upper bound is the total weight of all vertices. We use the following example to illustrate that the direct encoding cannot compute a tight upper bound even for a very simple MWC instance.

Example 1. The total weight of soft clauses of the direct encoding for the graph in Fig. 1 is 12 . Using unit propagation, $\left\{\left(x_{3}, 4\right),\left(x_{4}, 5\right)\right\}$ is found to be an inconsistent subset because of the hard clause $\bar{x}_{3} \vee \bar{x}_{4}$, then the upper bound can be improved by 4 . The left soft clauses are: $\left(x_{1}, 1\right)$, $\left(x_{2}, 2\right),\left(x_{4}, 1\right)$, then unit propagation detects that $\left\{\left(x_{1}, 1\right),\left(x_{4}, 1\right)\right\}$ is also inconsistent, then we can improve the upper bound by 1 . Finally, the upper bound is improved to 6, larger than the optimal solution 5.

Because of these drawbacks, the direct encoding does not perform well, as shown by the experimental results presented in Section 4.3. One might want to add an at-most-one constraint for each independent set to remedy the drawbacks of the direct encoding. That is, for each independent set $I=\left\{v_{1}, v_{2}, \ldots, v_{l}\right\}$, add the at-most-one constraint $x_{1}+x_{2}+\ldots+x_{l} \leq 1$. However, the at-most-one constraint does not add anything new, because the subset of hard clauses $\left\{\bar{x}_{i} \vee \bar{x}_{j} \mid 1 \leq i<j \leq l\right\}$ in the encoding already enforces the at-most-one constraint (Chen, 2010). In addition, the encoding of the 
at-most-one constraint using hard binary clauses is efficient enough when the independent set is not extremely large, which is usually the case when solving hard MC or MWC instances. Therefore, the at-most-one constraint is presumably useless in the encoding from MC or MWC into MaxSAT.

\subsubsection{Split Encoding And Iterative Split EnCoding of MWC into MaxSAT}

Another encoding is introduced for MC in MaxCLQ (Li \& Quan, 2010b) by defining the set of soft clauses based on an independent set partition of $G$. Concretely, MaxCLQ first partitions $G$ into a set of independent sets, then creates a soft clause for each independent set, which is a disjunction of the variables corresponding to the vertices in the independent set. The independent set based encoding is shown substantially more efficient than the direct encoding to solve MC.

A natural way to extend the independent set based MaxSAT encoding to MWC is to split vertex weights. For an independent set $I=\left\{v_{1}, v_{2}, \ldots, v_{l}\right\}$, where $w\left(v_{1}\right) \geq w\left(v_{2}\right) \geq \ldots \geq w\left(v_{l}\right)$, we can split vertex weights in $I$ by the minimum weight $w\left(v_{l}\right)$, thus we obtain a weighted soft clause $\left(x_{1} \vee x_{2} \vee \ldots \vee x_{l}, w\left(v_{l}\right)\right)$ and $l^{\prime}$ unit soft clauses: $\left(x_{1}, w\left(v_{1}\right)-w\left(v_{l}\right)\right),\left(x_{2}, w\left(v_{2}\right)-w\left(v_{l}\right)\right), \ldots$, $\left(x_{l^{\prime}}, w\left(v_{l^{\prime}}\right)-w\left(v_{l}\right)\right)$, where $l^{\prime}$ is the largest integer such that $w\left(v_{l^{\prime}}\right)>w\left(v_{l}\right)$ in $I$. This encoding is called the split encoding. The total weight of soft clauses in the split encoding should be less than that of the direct encoding, giving a better base upper bound, but it may still be large.

Example 2. A possible independent set partition of the graph in Fig. 1 is $\left\{v_{4}, v_{3}, v_{1}\right\}$ and $\left\{v_{2}\right\}$. The soft clauses of split encoding based on the partition are $\left(x_{4} \vee x_{3} \vee x_{1}, 1\right),\left(x_{4}, 4\right),\left(x_{3}, 3\right)$ and $\left(x_{2}, 2\right)$. The total weight of the soft clauses is 10 , which is better than that of the direct encoding. Using unit propagation, $\left\{\left(x_{4}, 4\right),\left(x_{3}, 3\right)\right\}$ is found to be an inconsistent subset because of the hard clause $\bar{x}_{3} \vee \bar{x}_{4}$, which allows us to improve the upper bound by 3 . In the same way, $\left\{\left(x_{4}, 1\right)\right.$, $\left.\left(x_{2}, 2\right)\right\}$ is found to be inconsistent, which improves the upper bound by 1 . Finally, the remaining soft clauses $\left(x_{4} \vee x_{3} \vee x_{1}, 1\right)$ and $\left(x_{2}, 1\right)$ are consistent. Thus, the upper bound computed using the split encoding is still 6.

The split encoding can be improved by the so-called the iterative split encoding, as used in MinSatz (Li et al., 2012). The idea of the iterative split encoding is to split vertex weights repeatedly until all vertices in the same independent set have the same weight. Concretely, for an independent set $I=\left\{v_{1}, v_{2}, \ldots, v_{l}\right\}$ such that $w\left(v_{1}\right) \geq w\left(v_{2}\right) \geq \ldots \geq w\left(v_{l}\right)$, we add a soft clause $\left(x_{1} \vee x_{2} \vee\right.$ $\left.\ldots \vee x_{l}, w\left(x_{l}\right)\right)$, and repeatedly find the largest $l^{\prime}$ such that $w\left(x_{l^{\prime}}\right)>w\left(x_{l}\right)$ and add a soft clause $\left(x_{1} \vee x_{2} \vee \ldots \vee x_{l^{\prime}}, w\left(x_{l^{\prime}}\right)-w\left(x_{l}\right)\right)$, while $l^{\prime} \geq 1$.

Example 3. A possible independent set partition of the graph in Fig. 1 is $\left\{v_{4}, v_{3}, v_{1}\right\},\left\{v_{2}\right\}$. The soft clauses of the iterative split encoding based on the partition are $\left(x_{4} \vee x_{3} \vee x_{1}, 1\right),\left(x_{4} \vee x_{3}, 3\right)$, $\left(x_{4}, 1\right)$ and $\left(x_{2}, 2\right)$. The total weight of soft clauses is 7 . Starting unit propagation by setting $x_{4}=1$, we find that $\left\{\left(x_{4}, 1\right),\left(x_{2}, 2\right)\right\}$ is an inconsistent subset, so that we can improve the upper bound by 1 and get a new soft clause $\left(x_{2}, 1\right)$. Then, we find that $\left\{\left(x_{4} \vee x_{3}, 3\right),\left(x_{2}, 1\right)\right\}$ is inconsistent, so the upper bound is improved to 5, which is the tightest upper bound.

The iterative split encoding gives a non-trivial base upper bound, which is better than those obtained by the direct encoding and the split encoding. The key point of the iterative split encoding is that the vertex weights are split in advance, generating numerous soft clauses, such that a variable may appear in several soft clauses and that some clauses may differ only in one variable (e.g. clauses $\left(x_{4} \vee x_{3} \vee x_{1}, 1\right)$ and $\left(x_{4} \vee x_{3}, 3\right)$ in Example 3). Many splittings may not be useful in the upper 
bound computation, and MaxSAT reasoning may be more complicated in such an instance with numerous soft clauses.

\subsubsection{LiterAL-Weighted EnCOding OF MWC INTO MAXSAT}

Recall that the MaxSAT encoding for MC in MaxCLQ guarantees that a variable appears only once in soft clauses and the number of soft clauses equals to the number of independent sets. MaxSAT reasoning in such an instance should be much simpler. In order to extend this advantage to MWC and not to split vertex weights in advance, we introduce the Literal-Weighted encoding (LW encoding) from MWC into LW MaxSAT, in which the literals in a soft clause are also weighted. Concretely, a weighted literal is a $\operatorname{pair}(\ell, w)$, where $\ell$ is a literal and $w$ is its weight. A literal-weighted soft clause (LW soft clause) is a disjunction of weighted literals. Formally, given an MWC instance $G=(V, E, w)$, we encode $G$ into an LW MaxSAT instance $\phi_{l w}$ as follows:

- Associate each vertex $v_{i}$ with a weighted literal $\left(x_{i}, w\left(x_{i}\right)\right)$, where $w\left(x_{i}\right)=w\left(v_{i}\right)$;

- Add a hard clause $\bar{x}_{i} \vee \bar{x}_{j}$ for each pair of unconnected vertices $v_{i}$ and $v_{j}$;

- For an independent set $I=\left\{v_{1}, v_{2}, \ldots, v_{l}\right\}$, add an LW soft clause $c=\left(x_{1}, w\left(v_{1}\right)\right) \vee\left(x_{2}, w\left(v_{2}\right)\right)$ $\vee \ldots \vee\left(x_{l}, w\left(v_{l}\right)\right)$.

The LW soft clause $c$ can also be presented as a set $\left\{\left(x_{1}, w\left(x_{1}\right)\right),\left(x_{2}, w\left(x_{2}\right)\right), \ldots,\left(x_{l}, w\left(x_{l}\right)\right)\right\}$. The weight of $c$ is defined to be

$$
w(c)=\max _{1 \leq j \leq l}\left(w\left(x_{j}\right)\right) .
$$

An LW clause $c$ is ordered if $w\left(x_{1}\right) \geq w\left(x_{2}\right) \geq \ldots \geq w\left(x_{l}\right)$. In the sequel, LW soft clauses are always ordered. Therefore, the weight of an LW soft clause is always $w\left(x_{1}\right)$, representing the cost if none of vertices in the corresponding independent set is included in the clique under construction. We now show that the optimal solution of an LW MaxSAT instance $\phi_{l w}$, denoted by $\operatorname{opt}\left(\phi_{l w}\right)$, which maximizes the total weight of satisfied soft clauses and satisfies all hard clauses, gives an upper bound of $\omega_{v}(G)$, differently from the usual encodings by which the optimal solution of a MaxSAT instance gives $\omega_{v}(G)$.

An MWC is given by an assignment of $\phi_{l w}$ that satisfies all hard clauses and maximizes the total weight of satisfied literals. Example 4 suggests that $\operatorname{opt}\left(\phi_{l w}\right)$ and $\omega_{v}(G)$ can be very different for an MWC instance $G$.

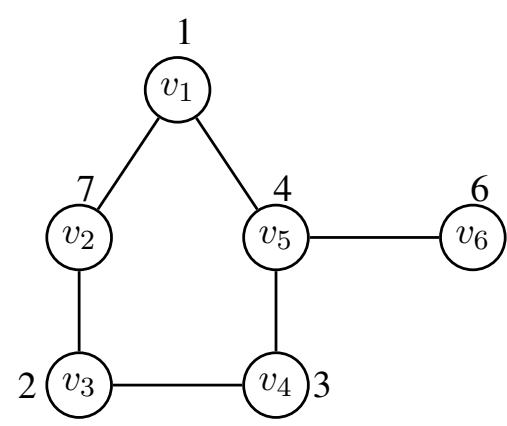

Figure 2: A weighted graph with 6 vertices and 6 edges. Numbers indicate vertex weights. 
Example 4. A possible independent set partition of the graph in Fig. 2 is $\left\{\left\{v_{1}, v_{3}, v_{6}\right\},\left\{v_{2}, v_{4}\right\}\right.$ and $\left.\left\{v_{5}\right\}\right\}$. The set of soft clauses of the LW MaxSAT instance $\phi_{l w}$ based on this partition is $\left\{\left\{\left(x_{6}\right.\right.\right.$, $\left.\left.6),\left(x_{3}, 2\right),\left(x_{1}, 1\right)\right\},\left\{\left(x_{2}, 7\right),\left(x_{4}, 3\right)\right\},\left\{\left(x_{5}, 4\right)\right\}\right\}$. The only MWC of the graph is $\left\{v_{5}, v_{6}\right\}$ and $\omega_{v}(G)=10$. On the other hand, $\left\{x_{1}=1, x_{2}=1\right\}$ is an optimal solution of $\phi_{l w}$ and $\operatorname{opt}\left(\phi_{l w}\right)=13$. Moreover, any optimal solution of $\phi_{l w}$ does not correspond to the maximum weight clique in the graph.

The following proposition states a relationship between the optimal solution of an LW MaxSAT instance and the MWC of the encoded graph.

Proposition 2. Consider a weighted graph $G=(V, E, w)$, let $\phi_{l w}$ be the $L W$ instance based on an independent set partition of $G$, then $\omega_{v}(G) \leq \operatorname{opt}\left(\phi_{l w}\right)$.

Proof. Suppose that $\left\{v_{i_{1}}, v_{i_{2}}, \ldots, v_{i_{p}}\right\}$ is a maximum weight clique of $G$. Let $c_{i_{j}}$ be the LW clause containing $x_{i_{j}}$. We have

$$
\omega_{v}(G)=\sum_{j=1}^{p} w\left(x_{i_{j}}\right) \leq \sum_{j=1}^{p} w\left(c_{i_{j}}\right) \leq \operatorname{opt}\left(\phi_{l w}\right) .
$$

Since $\operatorname{opt}\left(\phi_{l w}\right)$ is just an upper bound of $\omega_{v}(G)$, it makes little sense to apply a MaxSAT solver to find $\operatorname{opt}\left(\phi_{l w}\right)$. However, we can transform $\phi_{l w}$, so that an optimal solution of the new instance is a tighter upper bound of $\omega_{v}(G)$, and the upper bound of the new optimal solution can also be tightened. Example 5 illustrates how to do this.

Example 5. A possible independent set partition of the graph in Fig. 1 is $\left\{v_{4}, v_{3}, v_{1}\right\}$ and $\left\{v_{2}\right\}$. The $L W$ soft clauses are $\left\{\left(x_{4}, 5\right),\left(x_{3}, 4\right),\left(x_{1}, 1\right)\right\}$ and $\left\{\left(x_{2}, 2\right)\right\}$. The total weight of soft clauses is 7 , giving the base upper bound as in the iterative split encoding. Unit propagation by setting $x_{2}=1$ makes $x_{4}$ and $x_{3}$ false through the hard clauses. Then we find that we can split the clause $\left\{\left(x_{4}, 5\right)\right.$, $\left.\left(x_{3}, 4\right),\left(x_{1}, 1\right)\right\}$ into $\left\{\left(x_{4}, 2\right),\left(x_{3}, 2\right)\right\}$ and $\left\{\left(x_{4}, 3\right),\left(x_{3}, 2\right),\left(x_{1}, 1\right)\right\}$, and keep the base upper bound to be 7, but that the splitting allows to derive an inconsistent subset of soft clauses $\left\{\left\{\left(x_{4}, 2\right)\right.\right.$, $\left.\left.\left(x_{3}, 2\right)\right\},\left\{\left(x_{2}, 2\right)\right\}\right\}$ and to improve the base upper bound to 5, the tightest possible upper bound.

Note that unit propagation does not need to derive any empty clause to improve the upper bound in this example. Furthermore, in order to obtain the tightest upper bound, one unit propagation suffices, while two inconsistent subsets need to be derived in the iterative split encoding in Example 3.

The key point in Example 5 is that the original LW MaxSAT instance is transformed into a new one, of which the upper bound of the optimal solution is improved to 5 using MaxSAT reasoning. This tightest upper bound of the new LW MaxSAT instance is also the tightest upper bound of $\omega_{v}(G)$.

In the next subsection, we will define two sound transformation rules to transform an LW MaxSAT instance, allowing to derive a tight upper bound for MWC in general case. To be effective, the application of these two rules should be driven by MaxSAT reasoning, which will be presented in Subsection 3.4. 


\subsection{Transformation Rules for Literal-Weighted MaxSAT}

We propose two transformation rules to split an LW soft clause of an LW MaxSAT instance encoding an MWC instance $G$. The soundness of these rules is based on Proposition 3, ensuring that after splitting, an optimal solution of the new LW MaxSAT instance is a tighter upper bound of $\omega_{v}(G)$.

Proposition 3. Let $\phi_{l w}$ be an LW MaxSAT instance encoding of an MWC instance G. For any LW soft clause $c=\left\{\left(x_{1}, w\left(x_{1}\right)\right),\left(x_{2}, w\left(x_{2}\right)\right), \ldots,\left(x_{l}, w\left(x_{l}\right)\right)\right\}$ of $\phi_{l w}$ and $0<\delta \leq w\left(x_{1}\right)$, split $c$ into $c^{\prime}=\left\{\left(x_{1}, \delta\right),\left(x_{2}, \min \left(w\left(x_{2}\right), \delta\right)\right), \ldots,\left(x_{l}, \min \left(w\left(x_{l}\right), \delta\right)\right)\right\}$ and $c^{\prime \prime}=\left\{\left(x_{1}, w\left(x_{1}\right)-\delta\right),\left(x_{2}\right.\right.$, $\left.\left.\max \left(w\left(x_{2}\right)-\delta, 0\right)\right), \ldots,\left(x_{l}, \max \left(w\left(x_{l}\right)-\delta, 0\right)\right)\right\}$, where literals with weight 0 are removed, we get $\phi_{l w}^{\prime}=\left(\phi_{l w} \backslash\{c\}\right) \cup\left\{c^{\prime}, c^{\prime \prime}\right\}$, then $\omega_{v}(G) \leq \operatorname{opt}\left(\phi_{l w}^{\prime}\right) \leq \operatorname{opt}\left(\phi_{l w}\right)$.

Proof. First of all, note that both $c^{\prime}$ and $c^{\prime \prime}$ are ordered as $c$. To prove $\omega_{v}(G) \leq \operatorname{opt}\left(\phi_{l w}^{\prime}\right)$, we first show that $\min \left(w\left(x_{j}\right), \delta\right)+\max \left(w\left(x_{j}\right)-\delta, 0\right)=w\left(x_{j}\right)$ for any $j$ such that $1<j \leq l$. In fact,

1. If $w\left(x_{j}\right) \geq \delta, \min \left(w\left(x_{j}\right), \delta\right)+\max \left(w\left(x_{j}\right)-\delta, 0\right)=\delta+w\left(x_{j}\right)-\delta=w\left(x_{j}\right)$;

2. if $w\left(x_{j}\right)<\delta, \min \left(w\left(x_{j}\right), \delta\right)+\max \left(w\left(x_{j}\right)-\delta, 0\right)=w\left(x_{j}\right)+0=w\left(x_{j}\right)$.

In other words, the total weight of a literal is not changed by splitting $c$ into $c^{\prime}$ and $c^{\prime \prime}$. Let $C=$ $\left\{v_{i_{1}}, v_{i_{2}}, \ldots, v_{i_{p}}\right\}$ be a maximum weight clique of $G$. Then $\left\{x_{i_{1}}=1, x_{i_{2}}=1, \ldots, x_{i_{p}}=1\right\}$ with other variables being 0 , is an assignment of $\phi_{l w}^{\prime}$ satisfying all hard clauses. Let $S\left(x_{i_{j}}\right)=\left\{c \mid x_{i_{j}} \in\right.$ $c\}$ be the set of soft clauses containing $x_{i_{j}}$ of $\phi_{l w}^{\prime}$ for $1 \leq j \leq p$. All clauses in $S\left(x_{i_{j}}\right)$ are satisfied by $x_{i_{j}}$. At the same time,

$$
\sum_{c \in S\left(x_{i_{j}}\right)} w(c)=\sum_{c \in S\left(x_{i_{j}}\right)} \max _{x \in c} w(x) \geq w\left(x_{i_{j}}\right)
$$

Hence, we have,

$$
\operatorname{opt}\left(\phi_{l w}^{\prime}\right) \geq \sum_{j=1}^{p} \sum_{c \in S\left(x_{i_{j}}\right)} w(c) \geq \sum_{j=1}^{p} w\left(x_{i_{j}}\right)=\sum_{j=1}^{p} w\left(v_{i_{j}}\right)=\omega_{v}(G) .
$$

To prove $\operatorname{opt}\left(\phi_{l w}^{\prime}\right) \leq \operatorname{opt}\left(\phi_{l w}\right)$, let $\mathcal{A}$ be any assignment and $w\left(\phi_{l w}, \mathcal{A}\right)\left(w\left(\phi_{l w}^{\prime}, \mathcal{A}\right)\right)$ be the total weight of satisfied soft clauses of $\phi_{l w}\left(\phi_{l w}^{\prime}\right)$.

1. If $c$ is not satisfied in $\phi_{l w}$ by $\mathcal{A}$, then $c^{\prime}$ and $c^{\prime \prime}$ also are not satisfied in $\phi_{l w}^{\prime}$ by $\mathcal{A}$;

2. If $c$ is satisfied by $\mathcal{A}, c^{\prime}$ is also satisfied since $c^{\prime}$ has the same literals as $c$, but $c^{\prime \prime}$ may not be satisfied because the satisfied literal of $c$ may have weight 0 in $c^{\prime \prime}$ and may thus be removed from $c^{\prime \prime}$.

We note that $w(c)=w\left(c^{\prime}\right)+w\left(c^{\prime \prime}\right)$, so we have $w\left(\phi_{l w}, \mathcal{A}\right) \geq w\left(\phi_{l w}^{\prime}, \mathcal{A}\right)$ for any assignment $\mathcal{A}$, implying opt $\left(\phi_{l w}^{\prime}\right) \leq \operatorname{opt}\left(\phi_{l w}\right)$.

Given a weighted graph $G=(V, E, w)$, let $\phi_{l w}$ be an LW MaxSAT instance based on an independent set partition of $G$, we propose the following two rules to transform $\phi_{l w}$. 
1. $\delta$-Rule Given an LW soft clause $c=\left\{\left(x_{1}, w\left(x_{1}\right)\right),\left(x_{2}, w\left(x_{2}\right)\right), \ldots,\left(x_{l}, w\left(x_{l}\right)\right)\right\}$ of $\phi_{l w}$ and a weight $\delta$ such that $0<\delta \leq w\left(x_{1}\right)$, split $c$ into $c^{\prime}=\left\{\left(x_{1}, \delta\right),\left(x_{2}, \min \left(w\left(x_{2}\right), \delta\right)\right), \ldots,\left(x_{l}\right.\right.$, $\left.\left.\min \left(w\left(x_{l}\right), \delta\right)\right)\right\}$ and $c^{\prime \prime}=\left\{\left(x_{1}, w\left(x_{1}\right)-\delta\right),\left(x_{2}, \max \left(w\left(x_{2}\right)-\delta, 0\right)\right), \ldots,\left(x_{l}, \max \left(w\left(x_{l}\right)\right.\right.\right.$ $-\delta, 0))\}$, i.e., $\phi_{l w}^{\prime}=\left(\phi_{l w} \backslash\{c\}\right) \cup\left\{c^{\prime}, c^{\prime \prime}\right\}$.

2. (k, $\delta)$-Rule Given an LW clause $c=\left\{\left(x_{1}, w\left(x_{1}\right)\right),\left(x_{2}, w\left(x_{2}\right)\right), \ldots,\left(x_{l}, w\left(x_{l}\right)\right)\right\}$ of $\phi_{l w}$, an integer $1 \leq k<l$ and a weight $\delta$ such that $0<\delta \leq w\left(x_{1}\right)-w\left(x_{k+1}\right)$, split $c$ into $c^{\prime}=\left\{\left(x_{1}, \delta\right),\left(x_{2}\right.\right.$, $\left.\left.\max \left(w\left(x_{2}\right)+\delta-w\left(x_{1}\right), 0\right)\right), \ldots,\left(x_{k}, \max \left(w\left(x_{k}\right)+\delta-w\left(x_{1}\right), 0\right)\right)\right\}$, and $c^{\prime \prime}=\left\{\left(x_{1}, w\left(x_{1}\right)-\delta\right)\right.$, $\left(x_{2}, \min \left(w\left(x_{2}\right), w\left(x_{1}\right)-\delta\right)\right), \ldots,\left(x_{k}, \min \left(w\left(x_{k}\right), w\left(x_{1}\right)-\delta\right)\right),\left(x_{k+1}, w\left(x_{k+1}\right)\right), \ldots,\left(x_{l}\right.$, $\left.\left.w\left(x_{l}\right)\right)\right\}$, i.e., $\phi_{l w}^{\prime}=\left(\phi_{l w} \backslash\{c\}\right) \cup\left\{c^{\prime}, c^{\prime \prime}\right\}$.

The purpose of the $\delta$-Rule and the $(k, \delta)$-Rule is to split $c$ into a clause $c^{\prime}$ with weight $\delta$ and a clause $c^{\prime \prime}$ with weight $w\left(x_{1}\right)-\delta$ without changing the total weight of a literal. The constraint $\delta \leq w\left(x_{1}\right)-w\left(x_{k+1}\right)$ is to ensure that $c^{\prime \prime}$ remains ordered (i.e., $w\left(x_{1}\right)-\delta \geq \min \left(w\left(x_{2}\right), w\left(x_{1}\right)-\delta\right) \geq \ldots$ $\left.\left.\geq w\left(x_{k+1}\right)\right) \geq \ldots \geq w\left(x_{l}\right)\right)$. We use an example to illustrate the $(k, \delta)$-Rule. Let $c$ be an LW soft clause $c=\left\{\left(x_{1}, 5\right),\left(x_{2}, 3\right),\left(x_{3}, 2\right)\right\}$, (1) If $k=1$ and $\delta=2$, then $c^{\prime}=\left\{\left(x_{1}, 2\right)\right\}$ and $c^{\prime \prime}=\left\{\left(x_{1}, 3\right),\left(x_{2}\right.\right.$, $\left.3),\left(x_{3}, 2\right)\right\}$; (2) If $k=2$ and $\delta=3$, then $c^{\prime}=\left\{\left(x_{1}, 3\right),\left(x_{2}, 1\right)\right\}$ and $c^{\prime \prime}=\left\{\left(x_{1}, 2\right),\left(x_{2}, 2\right),\left(x_{2}, 2\right)\right\}$; (3) If $k=2$ and $\delta=2$, then $c^{\prime}=\left\{\left(x_{1}, 2\right),\left(x_{2}, 0\right)\right\}=\left\{\left(x_{1}, 2\right)\right\}$ and $c^{\prime \prime}=\left\{\left(x_{1}, 3\right),\left(x_{2}, 3\right),\left(x_{3}, 2\right)\right\}$.

The soundness of the $\delta$-Rule and the $(k, \delta)$-Rule can be easily proved using Proposition 3 . These two rules can be applied in many possible ways to generate many possible clauses. For example, as a special case, when $\delta=w\left(x_{l}\right)$ in the $\delta$-Rule, $\left.\left.\left.c^{\prime}=\left\{\left(x_{1}, \delta\right)\right),\left(x_{2}, \delta\right)\right), \ldots,\left(x_{l}, \delta\right)\right)\right\}$ and at least $x_{l}$ is removed from $c^{\prime \prime}$ since its weight is 0 in $c^{\prime \prime}$. In other words, we can transform $\phi_{l w}$ into a classical weighted MaxSAT instance by repeatedly applying the $\delta$-Rule with $\delta=w\left(x_{l}\right)$ to each clause $c$ containing literals with different weights. In this way, we can obtain a classical weighted partial MaxSAT instance $\varphi$, in which all literals in any soft clause have the same weight and whose optimal solution gives a maximum weight clique. Moreover, let $\phi_{i}$ be the MaxSAT instance obtained after $i$ applications of the $\delta$-Rule, we have $\operatorname{opt}\left(\phi_{l w}\right) \geq \operatorname{opt}\left(\phi_{1}\right) \geq \operatorname{opt}\left(\phi_{2}\right) \geq \ldots \geq \operatorname{opt}(\varphi)=\omega_{v}(G)$. In fact, $\varphi$ is an iterative split encoding of $G$.

Observe that each application of the $\delta$-Rule or the $(k, \delta)$-Rule gives a MaxSAT instance $\phi_{i}$ whose optimal solution gives a tighter upper bound of $\omega_{v}(G)$. Since unrestricted applications of the two rules may not be effective, we restrict their applications to the cases where an inconsistent subset of soft clauses with weight $\delta$ can be derived. Concretely, the $(k, \delta)$-Rule is always applied to a clause $c$ in which the $k$ most weighted literals are failed or falsified, allowing to obtain $c^{\prime}$ from which an inconsistent subset $S$ of soft clauses can be derived, and the $\delta$-Rule is always applied to a clause $c$ in an inconsistent subset of soft clauses. Each application allows to improve an upper bound of $\omega_{v}(G)$ by a positive weight $\delta$. Note that $\delta$ in both rules is strictly greater than 0 , because when $\delta=0$, both rules only generate new empty clauses with weight 0 , which cannot improve the upper bound. We call extended MaxSAT reasoning the application of the $\delta$-Rule and the $(k, \delta)$-Rule driven by MaxSAT reasoning in an LW MaxSAT instance. Details of our approach are given afterwards.

\subsection{Upper Bound Based on MaxSAT Reasoning}

In this section, we show the transformation of an LW MaxSAT instance driven by MaxSAT reasoning. In Subsection 3.4.1, we apply the $\delta$-Rule to transform an inconsistent subset of soft clauses. In Subsection 3.4.2 and Subsection 3.4.3, we introduce two notions, namely the Top- $k$ failed literal clause and the Top- $k$ empty clause, to deduce an inconsistent subset of soft clauses using the 
$(k, \delta)$-Rule, which is then transformed by applying the $\delta$-Rule. In Subsection 3.4.4, we present the overestimating algorithm that computes a tight upper bound for MWC by successively transforming an LW MaxSAT instance.

\subsubsection{Transforming an Inconsistent Subset of Soft Clauses}

First of all, we detect inconsistent subsets using unit propagation presented in Algorithm 1.

Example 6. A possible independent set partition of the graph in Fig. 2 is $\left\{\left\{v_{1}, v_{4}, v_{6}\right\},\left\{v_{2}\right.\right.$, $\left.\left.v_{5}\right\},\left\{v_{3}\right\}\right\}, L W$ soft clauses based on this partition are $c_{1}=\left\{\left(x_{6}, 6\right),\left(x_{4}, 3\right),\left(x_{1}, 1\right)\right\}, c_{2}=$ $\left\{\left(x_{2}, 7\right),\left(x_{5}, 4\right)\right\}$ and $c_{3}=\left\{\left(x_{3}, 2\right)\right\}$. When we set $x_{3}=1$ to satisfy the unit clause $c_{3}, \bar{x}_{3}$ will be removed from hard clauses $\bar{x}_{1} \vee \bar{x}_{3}, \bar{x}_{3} \vee \bar{x}_{5}, \bar{x}_{3} \vee \bar{x}_{6}$. Unit clauses $\bar{x}_{1}, \bar{x}_{5}$ and $\bar{x}_{6}$ imply that $x_{1}=0, x_{5}=0$ and $x_{6}=0$, respectively. So, both $c_{1}$ and $c_{2}$ become unit clauses. Accordingly, we have to set $x_{4}=1$ and $x_{2}=1$ to satisfy them, falsifying the hard clause $\bar{x}_{2} \vee \bar{x}_{4}$.

Consequently, an inconsistent soft clause subset $S=\left\{c_{1}, c_{2}, c_{3}\right\}$ is detected and the weight of the subset is 2 . Proposition 1 allows to decrease the upper bound by 2 , thus the improved upper bound is 13. Since all soft clauses are involved in the inconsistent subset, we cannot improve the upper bound any more. However, we can split the soft clauses in $S$ using the $\delta$-Rule based on the following proposition.

Proposition 4. Let $S=\left\{c_{1}, c_{2}, \ldots, c_{i}\right\}$ be an inconsistent subset of $L W$ soft clauses, if the $\delta$-Rule is applied to split every $c_{j} \in S$ into $c_{j}^{\prime}$ and $c_{j}^{\prime \prime}$ with $\delta=w(S)$, then $S^{\prime}=\left\{c_{1}^{\prime}, c_{2}^{\prime}, \ldots, c_{i}^{\prime}\right\}$ is still an inconsistent soft clause subset with weight $\delta$.

Proof. $S^{\prime}$ is clearly inconsistent, because the clauses in $S^{\prime}$ have the same literals as the clauses in $S$. In addition, every clause in $S^{\prime}$ has weight $\delta$. Hence, $S^{\prime}$ is an inconsistent subset of soft clauses with weight $\delta$.

The purpose of the splitting is to obtain $S^{\prime \prime}=\left\{c_{1}^{\prime \prime}, c_{2}^{\prime \prime}, \ldots, c_{i}^{\prime \prime}\right\}$ to further improve the upper bound.

Example 7. As presented in Example 6, $\left\{c_{1}, c_{2}, c_{3}\right\}$ is an inconsistent subset with weight 2. Applying $\delta$-Rule with $\delta=2$, we have $c_{1}^{\prime}=\left\{\left(x_{6}, 2\right),\left(x_{4}, 2\right),\left(x_{1}, 1\right)\right\}, c_{1}^{\prime \prime}=\left\{\left(x_{6}, 4\right),\left(x_{4}, 1\right)\right\}, c_{2}^{\prime}=\left\{\left(x_{2}, 2\right)\right.$, $\left.\left(x_{5}, 2\right)\right\}, c_{2}^{\prime \prime}=\left\{\left(x_{2}, 5\right),\left(x_{5}, 2\right)\right\}, c_{3}^{\prime}=\left\{\left(x_{3}, 2\right)\right\}$ and $c_{3}^{\prime \prime}=\left\{\left(x_{3}, 0\right)\right\}$ ( $c_{3}^{\prime \prime}$ is removed $)$.

It is easy to see that $\left\{c_{1}^{\prime}, c_{2}^{\prime}, c_{3}^{\prime}\right\}$ is an inconsistent subset of soft clauses with weight 2 , and that the remaining clauses $\left\{\left\{\left(x_{6}, 4\right),\left(x_{4}, 1\right)\right\},\left\{\left(x_{2}, 5\right),\left(x_{5}, 2\right)\right\}\right\}$ can be used to further improve the upper bound. However, unit propagation cannot be used to improve the upper bound any more because no unit clause exists. Moreover, failed literal detection does not work either because every soft clause contains at least one literal that is not failed. We propose to apply the $(k, \delta)$-Rule to split the Top-k literal failed clause defined afterwards to improve the upper bound.

\subsubsection{TOP-K FAILED Literal Detection}

Definition 1. An LW soft clause $c=\left\{\left(x_{1}, w\left(x_{1}\right)\right),\left(x_{2}, w\left(x_{2}\right)\right), \ldots,\left(x_{l}, w\left(x_{l}\right)\right)\right\}$ is Top- $k$ literal failed if $x_{1}, x_{2}, \ldots, x_{k}$ are all failed literals, where $1 \leq k<l$.

We define the Top- $k$ weight of an LW soft clause $c$ as $w_{k}(c)=w\left(x_{1}\right)-w\left(x_{k+1}\right)$, where $1 \leq$ $k<$ length $(c)$. 
Proposition 5. Consider an LW soft clause $c=\left\{\left(x_{1}, w\left(x_{1}\right)\right),\left(x_{2}, w\left(x_{2}\right)\right), \ldots,\left(x_{l}, w\left(x_{l}\right)\right)\right\}$. If $c$ is Top-k literal failed with $k<l$, and $I S\left(x_{j}\right)(1 \leq j \leq k)$ is the set of soft clauses making $x_{j}$ failed, split $c$ into $c^{\prime}$ and $c^{\prime \prime}$ using the $(k, \delta)$-Rule with $\delta=\min \left(w_{k}(c), w\left(I S\left(x_{1}\right)\right), w\left(I S\left(x_{2}\right)\right), \ldots, w\left(I S\left(x_{k}\right)\right)\right)$, then $\left\{c^{\prime}\right\} \cup I S\left(x_{1}\right) \cup I S\left(x_{2}\right) \cup \ldots \cup I S\left(x_{k}\right)$ is an inconsistent subset of soft clauses with weight $\delta$.

Proof. The set is clearly inconsistent, because the satisfaction of each literal in $c^{\prime}$ results in an empty clause in the set. The minimum weight of the clause in the set is $\delta$. So it is an inconsistent subset of soft clauses with weight $\delta$.

As soon as we determine that $c$ is Top- $k$ literal failed, we apply the $(k, \delta)$-Rule to split $c$ and use the $\delta$-Rule to split clauses in $I S\left(x_{1}\right) \cup I S\left(x_{2}\right) \cup \ldots \cup I S\left(x_{k}\right)$. An inconsistent subset with weight $\delta$ is obtained in this way. Observe that when $k=l,\{c\} \cup I S\left(x_{1}\right) \cup I S\left(x_{2}\right) \cup \ldots \cup I S\left(x_{l}\right)$ is a classical inconsistent subset of soft clauses with weight $\min \left(w(c), w\left(I S\left(x_{1}\right)\right), w\left(I S\left(x_{2}\right)\right), \ldots, w\left(I S\left(x_{l}\right)\right)\right)$ in which each clause is split only using the $\delta$-Rule.

Example 8. Consider the soft clauses produced in Example 7, $c_{1}=\left\{\left(x_{6}, 4\right),\left(x_{4}, 1\right)\right\}$ and $c_{2}=$ $\left\{\left(x_{2}, 5\right),\left(x_{5}, 2\right)\right\}$. We test $x_{2}$ in $c_{2}$ by setting $x_{2}=1$. To satisfy hard clauses $\bar{x}_{2} \vee \bar{x}_{6}$ and $\bar{x}_{2} \vee \bar{x}_{4}$, we need to set $x_{6}=0$ and $x_{4}=0$. Then, $c_{1}=\left\{\left(x_{6}, 4\right),\left(x_{4}, 1\right)\right\}$ becomes falsified, making $x_{2}$ failed. However, $x_{5}$ is not failed. Therefore $c_{2}$ is Top- $k$ literal failed with $k=1$. Applying the $(k, \delta)$-Rule with $k=1$ and $\delta=3$ to $c_{2}$, we have $c_{2}^{\prime}=\left\{\left(x_{2}, 3\right)\right\}$ and $c_{2}^{\prime \prime}=\left\{\left(x_{2}, 2\right),\left(x_{5}, 2\right)\right\}$. Applying the $\delta$-Rule with $\delta=3$ to $c_{1}$, we get $c_{1}^{\prime}=\left\{\left(x_{6}, 3\right),\left(x_{4}, 1\right)\right\}$ and $c_{1}^{\prime \prime}=\left\{\left(x_{6}, 1\right)\right\}$. Consequently, we get an inconsistent subset $\left\{c_{1}^{\prime}, c_{2}^{\prime}\right\}$ with weight 3 .

As a result, the detection of a Top-k literal failed clause allows to improve the upper bound by 3 , giving the tightest upper bound 10.

\subsubsection{Top-K Empty Clause Detection}

It is noteworthy that a literal can be declared to be failed, only if unit propagation of the literal falsifies all literals of a clause. Sometimes, the propagation of a literal $\ell$ just makes the most weighted literals in a soft clause $c$ falsified, but not all literals in $c$. In this case, $\ell$ cannot be declared to be failed, so we cannot improve the upper bound using approaches presented above. However, we can split $c$ using the $(k, \delta)$-Rule to obtain a falsified clause, so that $\ell$ can be declared to be failed.

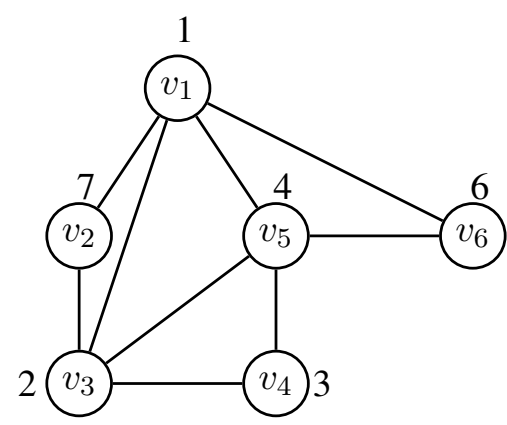

Figure 3: A weighted graph with 6 vertices and 9 edges. Numbers indicate vertex weight

Example 9. Consider the weighted graph $G$ in Fig. 3, a possible independent set partition of $G$ is $\left\{\left\{v_{6}, v_{3}\right\},\left\{v_{2}, v_{5}\right\},\left\{v_{4}, v_{1}\right\}\right\}$ and the $L W$ soft clauses are $c_{1}=\left\{\left(x_{6}, 6\right),\left(x_{3}, 2\right)\right\}, c_{2}=\left\{\left(x_{2}, 7\right)\right.$, 
$\left.\left(x_{5}, 4\right)\right\}$ and $c_{3}=\left\{\left(x_{4}, 3\right),\left(x_{1}, 1\right)\right\}$. The total weight of soft clauses of the LW MaxSAT instance based on this partition is 16 . On the one hand, the literals with the largest weight in each soft clause are not failed. When we set $x_{6}=1$, we need to set $x_{2}=0$ to satisfy the hard clause $\bar{x}_{2} \vee \bar{x}_{6}$. The literal $x_{6}$ is not failed, because unit propagation does not falsify any clause. However, the most weighted literal $x_{2}$ in $c_{2}$ is falsified. We can split $c_{2}$ into $c_{2}^{\prime}=\left\{\left(x_{2}, 3\right)\right\}$ and $c_{2}^{\prime \prime}=\left\{\left(x_{2}, 4\right),\left(x_{5}, 4\right)\right\}$. Then $c_{2}^{\prime}$ is falsified by propagating $x_{6}$. So, $x_{6}$ is a failed literal after splitting $c_{2}$, and $c_{1}$ is a Top- $k$ $(k=1)$ literal failed clause that can be split into $c_{1}^{\prime}=\left\{\left(x_{6}, 3\right)\right\}$ and $c_{1}^{\prime \prime}=\left\{\left(x_{6}, 3\right),\left(x_{3}, 2\right)\right\}$ using the $(k, \delta)$-Rule. We thus obtain an inconsistent subset $\left\{c_{1}^{\prime}, c_{2}^{\prime}\right\}$ with weight $\delta=3$. Consequently, the upper bound is improved by 3.

Example 9 suggests us to define the Top-k empty clause notion.

Definition 2. An LW soft clause $c=\left\{\left(x_{1}, w\left(x_{1}\right)\right),\left(x_{2}, w\left(x_{2}\right)\right), \ldots,\left(x_{l}, w\left(x_{l}\right)\right)\right\}$ is Top-k empty, where $1 \leq k<l$, if there is a literal $\ell$ such that when $\ell$ is assigned to true, unit propagation falsifies the literals $x_{1}, x_{2}, \ldots, x_{k}$ in $c$.

It is straightforward to show the following proposition.

Proposition 6. A literal can be declared to be failed after splitting a Top-k empty clause by using the $(k, \delta)$-Rule.

Proof. Splitting the Top- $k$ empty clause gives a clause $c=x_{1} \vee x_{2} \vee \ldots \vee x_{k}$, such that there is a literal $\ell$ of which the satisfaction makes $c$ empty via unit propagation.

\subsubsection{Overestimating Algorithm in MWCLQ}

Algorithm 3 formally describes how to successively transform an LW MaxSAT instance by applying the $\delta$-Rule and/or $(k, \delta)$-Rule to obtain disjoint inconsistent subsets of soft clauses in every search tree node of MWCLQ. The upper bound computed by Algorithm 3 in this way is very tight, as shown by our experimental results.

Given a weighted graph $G$, Algorithm 3 first encodes the MWC instance into an LW MaxSAT instance based on an independent set partition of $G$. The partitioning procedure works as follows. Vertices are sorted in the decreasing order of their weights (ties are broken in favor of the vertices with higher degree) and are successively inserted into an independent set. Suppose that the current independent sets are $I_{1}, I_{2}, \ldots, I_{i}$ (in this order, $i$ is 0 at the beginning of the partitioning process). The current first vertex $v$ is inserted into the the first $I_{j}$ such that $v$ is unconnected to all vertices already in $I_{j}$. If such a $I_{j}$ does not exist, a new independent set $I_{i+1}$ is opened and $v$ is inserted into $I_{i+1}$. Each independent set is encoded into an LW soft clause. The total weights of soft clauses is an initial upper bound for MWC of $G$ that should be improved by detecting disjoint inconsistent subsets of soft clauses using the extended MaxSAT reasoning.

The detection of an inconsistent subset of soft clauses is performed by detecting failed literals in the shortest available soft clause $c$. A literal is failed either because unit propagation falsifies all literals of another clause or just the most weighted literals of another soft clause. In the case all literals of $c$ are failed because unit propagation falsifies all literals of another clause, a usual inconsistent subset of soft clauses is obtained. In the case only the $k$ most weighted literals of $c$ are falsified and/or unit propagation of a literal of $c$ just falsifies the most weighted literals of another soft clause $t$, the $(k, \delta)$-Rule should be applied to split $c$ and/or $t$ to obtain an inconsistent subset of soft clauses. That is the reason why the Top- $k$ empty clauses and Top- $k$ literal failed clauses are 


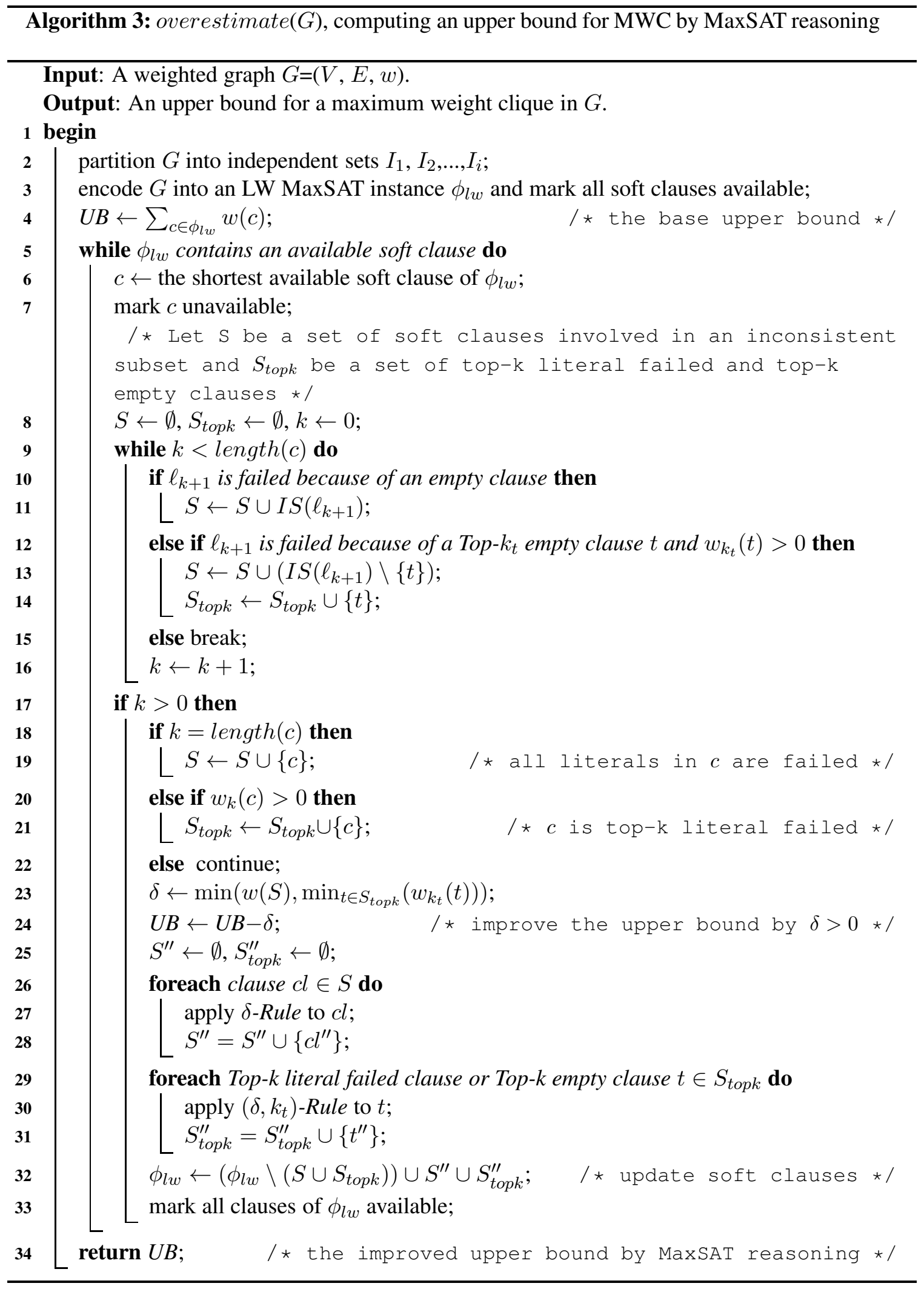


collected in $S_{\text {topk }}$. Meanwhile, the soft clauses involved in the inconsistent subset are collected in $S$. In any case, $\delta$ is computed as $\delta=\min \left(w(S), \min _{t \in S_{\text {topk }}}\left(w_{k_{t}}(t)\right)\right)$ (Line 23), where $w(S)$ is the minimum clause weight in $S$. Note that $S_{\text {topk }}$ is empty when $S$ is a usual inconsistent subset. The $\delta$-Rule and $(k, \delta)$-Rule are applied to split the clauses in the subset and the upper bound is improved by the weight of this subset.

Observe that Algorithm 3 is called at every search tree node from scratch because the graph at different search tree node is different. Also observe that the detected inconsistent subsets of soft clauses are disjoint, because a soft clause in one subset is not used to detect any other subset. In fact, an inconsistent subset of soft clauses is removed in Line 32 before detecting another inconsistent set. The initial upper bound is improved by the total weight of these inconsistent subsets. This approach might be further improved using MaxSAT resolution defined in the work of Bonet, Levy and, Manya (2006) and Larrosa, Heras, and Givry (2008), by adapting the approach of Abramé and Habet (2014) that transforms an inconsistent subset of clauses into a weighted empty clause and a set of new clauses that can be used in the detection of other inconsistent subsets of soft clauses. Nevertheless, the application of MaxSAT resolution has to be carefully driven for the approach to be competitive (Abramé \& Habet, 2015), because MaxSAT resolution produces many intermediate clauses.

We note that clauses produced using the $\delta$-Rule and the $(k, \delta)$-Rule cannot be obtained by simply applying MaxSAT resolution, because MaxSAT resolution transforms a MaxSAT instance into an equivalent one, while the $\delta$-Rule and the $(k, \delta)$-Rule may change the optimal solution of an LW MaxSAT instance by Proposition 3.

\section{Empirical Evaluation}

In this section, we empirically evaluate MWCLQ and its extended MaxSAT reasoning using standard benchmarks, namely the DIMACS benchmark, the BHOSLIB benchmark, random graphs, and the benchmark from the winner determination problem (WDP). We conducted four experiments in this study. The first experiment is to evaluate the performance of MWCLQ by comparing it with other state-of-the-art exact algorithms. The second experiment is to compare different encodings from MWC into MaxSAT. The third experiment is to investigate the impact of splitting soft clauses in the extended MaxSAT reasoning. In the forth experiment, we applied MWC algorithms to solve WDP instances and compare their performances.

We first introduce the benchmarks used in our experiments and describe the experimental environment. Then we present and discuss the experimental results in detail.

\subsection{Benchmarks and Experimental Environment}

Three types of benchmarks are used in our first three experiments.

1. DIMACS The DIMACS benchmark is taken from the Second DIMACS Implementation Challenge, which has been used widely for benchmarking purposes in the literature of algorithms for MC, MWC, MVC, MIS and so on. The 80 DIMACS instances are generated from real-world applications such as coding theory, fault diagnosis, Keller's conjecture and the Steiner Triple Problem, as well as random graphs generated with different properties, such as the DSJC, brock and p_hat families. The size of these instances ranges from less than $\mathbf{5 0}$ 
vertices and 1,000 edges to more than 4,000 vertices and 5,000,000 edges. We downloaded all instances from a website (ThanhVu \& Thang, 2014).

2. BHOSLIB BHOSLIB (Xu, 2004)(Benchmarks with Hidden Optimum Solutions for Graph Problems) instances are based on a CSP model named RB (Xu \& Li, 2000; Xu, Boussemart, Hemery, \& Lecoutre, 2007). Phase transitions exist for model RB and the transition points can be located exactly. BHOSLIB instances are generated in the phase transition region of model RB and appear to be extremely hard to solve for various algorithms, even when the graph size is small (Liu, Lin, Wang, Su, \& Xu, 2011; Xu \& Li, 2006). They were firstly used in the SAT competition 2004, and then have been widely used to evaluate algorithms for MC, MVC and MIS.

3. Random A random graph of $n$ vertices and density $p$ is generated by randomly selecting each edge with probability $p$ from the complete graph of $n$ vertices. In our experiments, $n$ ranges from 150 to 700 and $p$ from 0.5 to 0.95 . Random graphs allow to show the asymptotic behavior of an algorithm.

We convert a non-weighted graph into a weighted graph by associating a weight $w\left(v_{i}\right)=i \bmod$ $200+1$ to each vertex $v_{i}$. This method was initially proposed by Pullan (2008) and has been used as a standard converting approach to generate weighted graphs from non-weighted instances (Wu et al., 2012; Benlic \& Hao, 2013).

All solvers used in our experiments are implemented in $C / C++$. We compile them using $g c c / g++4.7 .2$ with option "-O3". All experiments are running on a machine with Intel(R) Xeon(R) CPU E5-2680 @ 2.70GHz, 8 cores and 16G RAM under Debian GNU/Linux 7.4. The cut-off time for a solver to solve an instance is one hour (3600 seconds).

\subsection{Comparison of MWCLQ with Other Algorithms}

We compare MWCLQ with two state-of-the-art exact solvers specific for MWC, a MinSAT solver and CPLEX.

1. Cliquer is a state-of-the-art solver for both MC problem and MWC problem. To our best knowledge, almost all recent exact algorithms for MWC (e.g., Kumlander, 2004; Shimizu et al., 2013, 2012) are based on Cliquer. We used the latest version of Cliquer released in 2010, available at its homepage (Östergård, 2010).

2. DKum (Kumlander, 2004, 2008b) is implemented in $V B$. The source code can be found at the author's homepage (Kumlander, 2008a). To execute it in our experimental environment, we translated it into $C$.

3. MinSatz (Li et al., 2012) is an exact weighted partial MinSAT solver, which achieves the state-of-the-art performance on solving clique problems and combinatorial auction problems.

4. CPLEX is a high-performance mathematical programming solver for linear programming, mixed integer programming, quadratic programming, and quadratically constrained programming problems. Let $G=(V, E, w)$ be a weighted graph, where $V=\left\{v_{1}, v_{2}, \ldots, v_{n}\right\}$, the 
integer programming formulation of the MWC instance $G$ is usually defined as follows.

$$
\max \sum_{i=1}^{n} x_{i} * w\left(v_{i}\right)
$$

subject to

$$
\begin{aligned}
x_{i}+x_{j} \leq 1, & \forall\left\{v_{i}, v_{j}\right\} \notin E \\
x_{i} \in\{0,1\}, & i=1,2, \ldots, n .
\end{aligned}
$$

Observe that the at-most-one constraint is enforced for every independent set of $G$. A solution of the integer programming problem corresponds to a maximum weight clique of $G$. CPLEX 12.6 was used in our experiments to solve MWC after encoding it as an integer programming problem in this way.

Table 1 shows runtimes of different solvers on DIMACS and BHOSLIB benchmarks. All 80 instances from the DIMACS benchmark are used in this experiment. MWCLQ solves 61 instances within the cut-off time, while Cliquer, DKum, MinSatz and CPLEX solves 52, 48, 58 and 44 instances, respectively. For simplicity, we exclude the instances solved within 100 seconds by all solvers or not solved by any solver within 3600 seconds. For the 39 instances displayed in Table 1, MWCLQ outperforms Cliquer on 32 instances and is comparable with Cliquer on other instances. MWCLQ significantly outperforms DKum on all instances except hamming10-2, which can be solved by both within 20 seconds. MWCLQ dominates MinSatz on 32 instances. In particular, for the 8 instances from the brock family, MWCLQ is about $20 X$ faster than MinSatz for the instances with 400 vertices and solves all four instances with 800 vertices, which cannot be solved by MinSatz. MWCLQ outperforms CPLEX on 28 instances. Particularly, CPLEX cannot solve any instance from the brock and $p$ hat families, while MWCLQ can solve all of them efficiently. It is interesting that both MinSatz and CPLEX solve MANN_a27 and MANN_a45 within the cut-off time, which cannot be solved by any specific MWC solver. Note that MANN_a27 and MANN_a45 are also hard for heuristic algorithms. For instance, BLS (Benlic \& Hao, 2013) can only find an approximating solution of 12281 with the success rate $16 \%$ within 396.58 seconds for MANN_a27, while PLS (Pullan, 2008) can only find an approximating solution of 12264.

We also used all 40 instances from the BHOSLIB benchmark. These instances are extremely hard for exact MWC solvers. All evaluated solvers can solve at most the 5 smallest instances with 450 vertices. Both MWCLQ and DKum solve 5 instances, CPLEX, Cliquer and MinSatz solves 3, 1 and 0 instances, respectively. Moreover, MWCLQ achieves the best performance on 4 out of the 5 instances.

Table 2 shows mean runtimes on random graphs. We generate 50 graphs at each point. MWCLQ is the only algorithm which solves all 700 graphs in this experiment within the cutoff time, while Cliquer, Dkum, MinSatz and CPLEX solves 595, 548, 548 and 387 instances, respectively. MWCLQ significantly outperforms Dkum and MinSatz on all instances and dominates CPLEX at all points except $(200,0.95)$ where both algorithms solve all instances within 30 seconds. MWCLQ dominates Cliquer completely on random graphs with density $D \geq 0.7$, and is comparable with Cliquer when $D<0.7$. MWCLQ is the only solver which can solve all instances at the point $(700,0.7)$. The experimental results suggest in particular that MWCLQ is very effective in solving graphs with 
Table 1: Runtimes (in seconds) on DIMACS and BHOSLIB benchmarks. The cut-off time is 3600 seconds. $|V|$ stands for the number of vertices, $D$ for the density of the graph and $\omega_{v}$ for the optimal solution of MWC. When a solver cannot solve an instance within 3600 seconds, its runtime is marked by '-'. Instances solved within 100 seconds by all solvers or not solved by any solver within the cut-off time are omitted.

\begin{tabular}{|c|c|c|c|c|c|c|c|c|}
\hline Graph & $|V|$ & $\mathrm{D}$ & $\omega_{v}$ & Cliquer & DKum & MinSatz & CPLEX & MWCLQ \\
\hline brock400_1 & 400 & 74 & 3422 & 260.2 & 607.2 & 2046 & - & 129.3 \\
\hline brock400_2 & 400 & 75 & 3350 & 366.1 & 524.5 & 2737 & - & 127.7 \\
\hline brock400_3 & 400 & 74 & 3471 & 290.7 & 573.6 & 2363 & - & 107.2 \\
\hline brock400_4 & 400 & 75 & 3626 & 287.4 & 606.3 & 1609 & - & 81.81 \\
\hline brock800_1 & 800 & 65 & 3121 & 1548 & - & - & - & 1427 \\
\hline brock800_2 & 800 & 65 & 3043 & 1603 & - & - & - & 2002 \\
\hline brock800_3 & 800 & 65 & 3076 & 1702 & - & - & - & 1545 \\
\hline brock800_4 & 800 & 65 & 2971 & 1990 & - & - & - & 2039 \\
\hline C250.9 & 250 & 89 & 5092 & - & 2215 & 640.6 & 30.41 & 39.99 \\
\hline DSJC1000.5 & 1000 & 50 & 2186 & 32.50 & 91.88 & 3527 & - & 81.30 \\
\hline DSJC500.5 & 500 & 50 & 1725 & 0.97 & 0.97 & 32.80 & - & 0.92 \\
\hline gen200_p0.9_44 & 200 & 89 & 5043 & 678.6 & 119.8 & 72.40 & 2.44 & 7.00 \\
\hline gen200_p0.9_55 & 200 & 89 & 5416 & 1718 & 362.2 & 35.19 & 1.81 & 2.76 \\
\hline gen400_p0.9_75 & 400 & 90 & 8006 & - & - & - & 238.7 & - \\
\hline hamming10-2 & 1024 & 99 & 50512 & 1469 & 7.47 & - & 0.06 & 15.46 \\
\hline johnson32-2-4 & 496 & 88 & 2033 & - & - & - & 0.43 & - \\
\hline MANN_a27 & 378 & 98 & 12283 & - & - & 4.45 & 2.07 & - \\
\hline MANN_a45 & 1035 & 99 & 34265 & - & - & 3487 & 32.61 & - \\
\hline p_hat1000-1 & 1000 & 24 & 1514 & 0.12 & 0.35 & 21.75 & - & 0.53 \\
\hline p_hat 1000-2 & 1000 & 49 & 5777 & - & - & 1628 & - & 2501 \\
\hline p_hat1500-1 & 1500 & 25 & 1619 & 1.03 & 2.72 & 252.3 & - & 4.12 \\
\hline p_hat500-1 & 500 & 25 & 1231 & 0.02 & 0.02 & 1.52 & - & 0.01 \\
\hline p_hat500-2 & 500 & 50 & 3920 & 5.59 & 3.86 & 10.41 & - & 2.34 \\
\hline p_hat500-3 & 500 & 75 & 5375 & - & - & 1009 & - & 916.7 \\
\hline p_hat700-1 & 700 & 25 & 1441 & 1.03 & 0.17 & 4.42 & - & 0.13 \\
\hline p_hat700-2 & 700 & 50 & 5290 & 169.5 & 213.4 & 39.93 & - & 47.88 \\
\hline san 1000 & 1000 & 50 & 1716 & - & - & 46.90 & - & 183.8 \\
\hline san200_0.7_2 & 200 & 69 & 2422 & 403.9 & 23.28 & 0.11 & 0.70 & 0 \\
\hline san200_0.9_1 & 200 & 89 & 6825 & - & 166.9 & 0.10 & 0.31 & 0.24 \\
\hline san200_0.9_2 & 200 & 89 & 6082 & 223.3 & 15.11 & 16.37 & 0.83 & 1.64 \\
\hline san200_0.9_3 & 200 & 89 & 4748 & 2329 & 470.3 & 118.2 & 3.54 & 16.19 \\
\hline san400_0.7_1 & 400 & 70 & 3941 & - & - & 12.62 & 24.45 & 3.44 \\
\hline san400_0.7_2 & 400 & 70 & 3110 & - & - & 34.74 & 87.75 & 4.98 \\
\hline san400_0.7_3 & 400 & 70 & 2771 & - & - & 96.12 & 43.53 & 6.37 \\
\hline san400_0.9_1 & 400 & 90 & 9776 & - & - & 1849 & 13.30 & 1257 \\
\hline sanr200_0.7 & 200 & 69 & 2325 & 0.20 & - & 2.73 & 10.81 & 0.13 \\
\hline sanr200_0.9 & 200 & 89 & 5126 & 1150 & 215.3 & 68.97 & 11.01 & 6.40 \\
\hline sanr400_0.5 & 400 & 50 & 1835 & 0.15 & - & 9.44 & - & 0.31 \\
\hline sanr400_0.7 & 400 & 70 & 2992 & 29.98 & 74.48 & 429.0 & - & 25.57 \\
\hline \multicolumn{4}{|c|}{ Total: 80} & 52 & 48 & 58 & 44 & 61 \\
\hline rb30-15-1 & 450 & 82 & 2990 & - & 177.7 & - & - & 244.9 \\
\hline frb30-15-2 & 450 & 82 & 3006 & 1065 & 44.98 & - & - & 30.14 \\
\hline frb30-15-3 & 450 & 82 & 2995 & - & 423.4 & - & 193.5 & 131.7 \\
\hline frb30-15-4 & 450 & 82 & 3032 & - & 287.5 & - & 258.2 & 181.8 \\
\hline frb30-15-5 & 450 & 82 & 3011 & - & 154.0 & - & 118.3 & 57.31 \\
\hline \multicolumn{4}{|c|}{ Total: 40} & 1 & 5 & 0 & 3 & 5 \\
\hline
\end{tabular}


Table 2: Mean runtimes in seconds on random graphs, obtained by solving 50 graphs at each point. The cut-off time is 3600 seconds. $|V|$ stands for the number of vertices, $D$ for the density of the graph and $\bar{\omega}_{v}$ for the weight of an optimal solution, averaged over the solved instances. The mean runtime is marked by '-' if no instance is solved at the point within the cut-off time. \# stands for the number of instances solved by the solver within the cut-off time

\begin{tabular}{|c|c|c|c|c|c|c|c|c|c|c|c|c|}
\hline \multicolumn{3}{|c|}{ Benchmark } & \multicolumn{2}{|c|}{ Cliquer } & \multicolumn{2}{|c|}{ DKum } & \multicolumn{2}{|c|}{ MinSatz } & \multicolumn{2}{|c|}{ CPLEX } & \multicolumn{2}{|c|}{ MWCLQ } \\
\hline$|V|$ & D & $\bar{\omega}_{v}$ & Time & \# & Time & \# & Time & \# & Time & \# & Time & \# \\
\hline 150 & 0.90 & 3394 & 21.06 & 50 & 7.92 & 50 & 3.57 & 50 & 2.58 & 50 & 0.58 & 50 \\
\hline 150 & 0.95 & 4766 & 1006 & 50 & 35.42 & 50 & 2.21 & 50 & 1.99 & 50 & 0.42 & 50 \\
\hline 200 & 0.80 & 3249 & 4.02 & 50 & 5.05 & 50 & 12.65 & 50 & 54.83 & 50 & 0.99 & 50 \\
\hline 200 & 0.90 & 5095 & 974.9 & 50 & 373.0 & 50 & 94.63 & 50 & 23.45 & 50 & 14.30 & 50 \\
\hline 200 & 0.95 & 7372 & - & 0 & 2464 & 19 & 111.1 & 50 & 3.91 & 50 & 28.77 & 50 \\
\hline 300 & 0.70 & 2441 & 1.76 & 50 & 3.01 & 50 & 32.36 & 50 & 353.5 & 50 & 1.13 & 50 \\
\hline 300 & 0.80 & 3334 & 81.45 & 50 & 107.1 & 50 & 379.6 & 50 & 303.7 & 47 & 14.87 & 50 \\
\hline 300 & 0.90 & 5351 & - & 0 & - & 0 & - & 0 & 263.5 & 40 & 845.4 & 50 \\
\hline 500 & 0.60 & 2285 & 3.94 & 50 & 9.45 & 50 & 208.5 & 50 & - & 0 & 6.03 & 50 \\
\hline 500 & 0.70 & 2969 & 119.9 & 50 & 302.1 & 50 & 2994 & 48 & - & 0 & 93.36 & 50 \\
\hline 600 & 0.60 & 2496 & 23.51 & 50 & 62.46 & 50 & 1006 & 50 & - & 0 & 34.79 & 50 \\
\hline 600 & 0.70 & 3293 & 1256 & 50 & 2884 & 29 & - & 0 & - & 0 & 869.9 & 50 \\
\hline 700 & 0.60 & 2510 & 46.01 & 50 & 132.8 & 50 & 2757 & 50 & - & 0 & 78.24 & 50 \\
\hline 700 & 0.70 & 3283 & 3006 & 45 & - & 0 & - & 0 & - & 0 & 2434 & 50 \\
\hline \multicolumn{3}{|c|}{ Total 700 } & \multicolumn{2}{|c|}{595} & \multicolumn{2}{|c|}{548} & \multicolumn{2}{|c|}{548} & \multicolumn{2}{|c|}{387} & \multicolumn{2}{|c|}{700} \\
\hline
\end{tabular}

Table 3: Lower bounds given by different solvers for DIMACS instances which cannot be solved by any solver within the cut-off time.

\begin{tabular}{|c|c|c|c|c|c|c|c|}
\hline Instance & $|V|$ & $\mathrm{D}$ & Cliquer & DKum & MinSatz & CPLEX & MWCLQ \\
\hline C1000.9 & 1000 & 90 & 1181 & 1846 & 6385 & 8066 & $\mathbf{8 4 7 1}$ \\
C2000.5 & 2000 & 50 & $\mathbf{2 4 6 6}$ & 1186 & 2198 & 1358 & $\mathbf{2 4 6 6}$ \\
C2000.9 & 2000 & 90 & 534 & 782 & 6747 & 7362 & $\mathbf{1 0 0 3 4}$ \\
C4000.5 & 4000 & 50 & 1284 & 863 & 2263 & 1854 & $\mathbf{2 6 9 8}$ \\
C500.9 & 500 & 90 & 1868 & 2070 & 5594 & 6520 & $\mathbf{6 6 7 2}$ \\
MANN_a81 & 3321 & 100 & 195 & 1201 & 100970 & $\mathbf{1 1 1 3 8 6}$ & 111033 \\
gen400_p0.9_55 & 400 & 90 & 2501 & 3037 & 5988 & 6611 & $\mathbf{6 6 7 6}$ \\
gen400_p0.9_65 & 400 & 90 & 2855 & 3179 & 6180 & 6720 & $\mathbf{6 8 3 2}$ \\
hamming10-4 & 1024 & 83 & 738 & 1798 & 4062 & $\mathbf{5 0 4 2}$ & 4614 \\
keller5 & 776 & 75 & 2860 & 2139 & $\mathbf{3 3 1 7}$ & $\mathbf{3 3 1 7}$ & $\mathbf{3 3 1 7}$ \\
keller6 & 3361 & 82 & 511 & 1637 & 5595 & $\mathbf{6 9 3 7}$ & 6316 \\
p_hat1000-3 & 1000 & 74 & 2417 & 2893 & 6779 & 7258 & $\mathbf{7 5 8 8}$ \\
p_hat1500-2 & 1500 & 51 & 2897 & 3127 & 6100 & 5954 & $\mathbf{7 1 0 4}$ \\
p_hat1500-3 & 1500 & 75 & 1521 & 2067 & 7050 & $\mathbf{9 0 5 8}$ & 8449 \\
p_hat700-3 & 700 & 75 & 2822 & 5156 & 7340 & 7400 & $\mathbf{7 5 6 5}$ \\
\hline
\end{tabular}




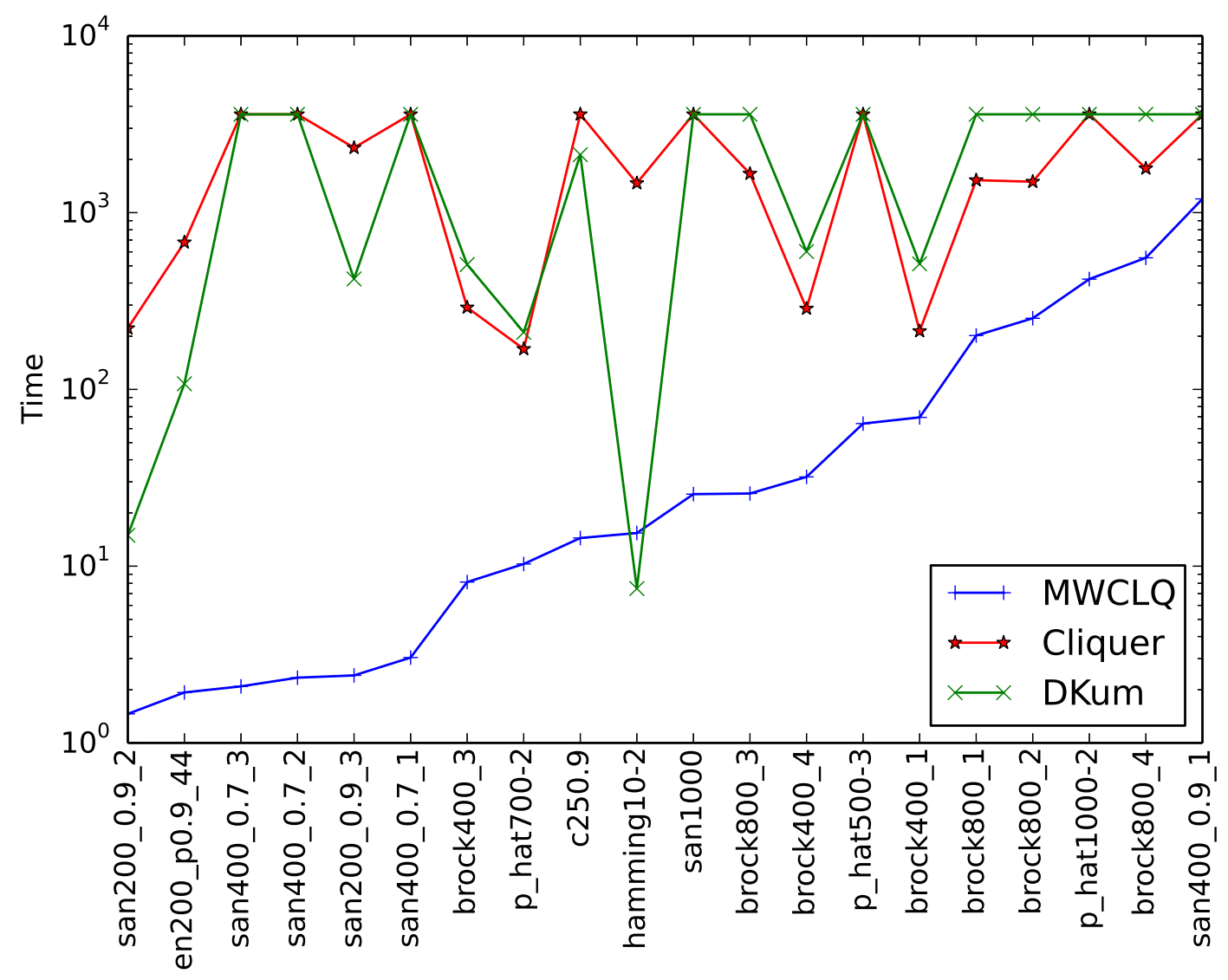

Figure 4: Runtimes to find an optimal solution. Instances for which all solvers find an optimal solution within 100 seconds or MWCLQ finds an optimal solution in less than one second are omitted

high density. CPLEX is also effective for dense graphs, but it cannot solve any random instance with more than 500 vertices.

For the 15 DIMACS instances that cannot be solved by any algorithm within the cut-off time, we report the largest weight clique found by each solver before the algorithm terminates, which is a lower bound of the optimal solution. Table 3 shows MWCLQ computes the best lower bounds for 11 instances, while CPLEX gives the best lower bounds for 5 instances. MWCLQ can give a significantly better lower bound than other MWC solvers on all instances except $C 2000.5$, where MWCLQ and Cliquer share the same bound. This result suggests that MWCLQ can often compute a better approximate solution than other exact solvers within a given time.

An exact algorithm for MC or MWC usually solves an instance in two phases. In the first phase, the algorithm finds an optimal solution. Then in the second phase, the algorithm proves that the solution is indeed optimal by showing that no better solution exists. In Fig. 4, we compare the runtimes that Cliquer, Dkum and MWCLQ need to find an optimal solution of a DIMACS instance. 
For simplicity, the instances for which all solvers find an optimal solution within 100 seconds or MWCLQ finds an optimal solution in less than one second are omitted. MWCLQ always finds an optimal solution much faster than other solvers on all instances except hamming10-2. For few instances such as brock800_2 and brock800_4, although MWCLQ spends more time than Cliquer for exactly solving them (see Table 1), it finds an optimal solution 10 times faster than Cliquer.

In summary, both Table 3 and Fig. 4 show that MWCLQ generally finds an optimal solution much faster than other solvers, although sometimes it may spend more time to prove the optimality.

\subsection{Comparison of Different Encodings of MWC into MaxSAT}

We presented four encodings from MWC into MaxSAT, namely the direct encoding, the split encoding, the iterative split encoding and the LW encoding. When an MWC instance is encoded into a MaxSAT instance using the first three encodings, a MaxSAT solver can be used to search for an optimal solution of the MWC instance. However, if the MWC instance is encoded into a MaxSAT instance using the LW encoding, the optimal solution of the MaxSAT instance is not an MWC, but an upper bound for the MWC. Therefore, when a MaxSAT solver is used to solve an MWC instance, only the first three encodings can be used.

Different from MaxSAT solvers, MWCLQ uses MaxSAT reasoning just in the upper bounding procedure at each search tree node, where the current subgraph is dynamically encoded into a MaxSAT instance. So, all the four encodings could be used in MWCLQ to encode the current subgraph.

Recall that MWCLQ is based on the LW encoding. We implemented three other versions of MWCLQ, i.e., MWCLQ ${ }_{d i r}$, MWCLQ $s p$ and MWCLQ $i t$, which are identical to MWCLQ, but are based on the direct encoding, the split encoding and the iterative split encoding, respectively. In a MaxSAT instance obtained using the direct encoding, all inconsistent subsets only contain two soft clauses, because all soft clauses are unit. When MWCLQ $_{\text {dir }}$ detects an inconsistent subset such as $\left\{\left(x_{1}, w\left(x_{1}\right)\right),\left(x_{2}, w\left(x_{2}\right)\right)\right\}$, the most weighted clause in the subset, say, $\left(x_{2}, w\left(x_{2}\right)\right)$, is split into $\left(x_{2}, w\left(x_{1}\right)\right)$ and $\left(x_{2}, w\left(x_{2}\right)-w\left(x_{1}\right)\right)$. Then the upper bound is improved by $w\left(x_{1}\right)$, and the clause $\left(x_{2}, w\left(x_{2}\right)-w\left(x_{1}\right)\right)$ can be used for further detection. In a MaxSAT instance obtained using the split and the iterative split encoding, all vertices in the same soft clause have the same weight. Observe that the Top- $k$ literal failed clause and the Top- $k$ empty clause do not make sense, and the $(k, \delta)$-Rule is not needed in the three encoding.

In this experiment, we ran the following four different kinds of state-of-the-art MaxSAT solvers to solve the MWC instances encoded into MaxSAT using the direct encoding, the split encoding and the iterative split encoding.

1. akMaxSat (Kügel, 2010) is a branch-and-bound MaxSAT solver that computes the lower bound with a combination of MaxSAT resolution and detection of disjoint inconsistent subsets. One of its authors sent us the source code submitted to the MaxSAT evaluation 2012.

2. MaxSatz (Li et al., 2007) is a branch-and-bound MaxSAT solver that incorporates some SAT technologies. We used the latest version MaxSatz2013, which is one of the best solvers in the MaxSAT evaluation 2013.

3. WPM1-2013 (Ansótegui, Bonet, \& Levy, 2009) is a MaxSAT solver based on successive calls to a SAT solvers. One of its authors provided us an executable file of WPM which is used in the MaxSAT evaluation 2013. 
4. MaxHS (Davies \& Bacchus, 2013b) is a hybrid Maxsat solver that exploits both SAT and integer programming technologies.

We report the number of instances solved by MWCLQ dir $_{\text {, MWCLQ }}$, MWCLQ MW $_{i t}$ MWCLQ, akMaxSat, MaxSatz and MaxHS within 3600 seconds. The results of WPM are not reported, because it solves only 3 DIMACS instances and does not solve any BHOSLIB instance. In addition, it always runs out of memory when solving random instances.

Table 4: Number of instances solved within 3600 seconds by MaxSAT solvers using three different encodings and four versions of MWCLQ. Direct stands for the direct encoding, Split is for the split encoding and Iter is for the iterative split encoding.

\begin{tabular}{|c|c|c|c|c|c|c|c|c|c|c|c|c|c|c|}
\hline \multicolumn{2}{|c|}{ Instance } & \multicolumn{3}{|c|}{ akMaxSat } & \multicolumn{3}{|c|}{ MaxSatz } & \multicolumn{3}{|c|}{ MaxHS } & \multirow{2}{*}{$\begin{array}{l}\text { MWC- } \\
\mathrm{LQ}_{\text {dir }}\end{array}$} & \multirow{2}{*}{$\begin{array}{l}\text { MWC- } \\
\mathrm{LQ}_{s p}\end{array}$} & \multirow{2}{*}{$\begin{array}{c}\mathrm{MWC}- \\
\mathrm{LQ}_{i t}\end{array}$} & \multirow{2}{*}{$\begin{array}{c}\text { MWC } \\
\text { LQ }\end{array}$} \\
\hline Benchmark & \# & Direct & Split & Iter & Direct & Split & Iter & Direct & Split & Iter & & & & \\
\hline brock & 12 & 4 & 4 & 4 & 4 & 4 & 4 & 4 & 0 & 0 & 4 & 8 & 8 & 12 \\
\hline c-fat & 7 & 7 & 7 & 7 & 7 & 7 & 7 & 7 & 7 & 7 & 7 & 7 & 7 & 7 \\
\hline $\mathrm{C}$ & 7 & 2 & 2 & 2 & 2 & 2 & 2 & 2 & 1 & 1 & 1 & 2 & 2 & 2 \\
\hline DSJC & 2 & 0 & 0 & 0 & 1 & 1 & 1 & 0 & 0 & 0 & 2 & 2 & 2 & 2 \\
\hline gen & 5 & 2 & 2 & 2 & 2 & 2 & 2 & 3 & 0 & 0 & 0 & 2 & 2 & 2 \\
\hline hamming & 6 & 5 & 5 & 5 & 5 & 5 & 5 & 5 & 4 & 3 & 3 & 4 & 4 & 5 \\
\hline johnson & 4 & 3 & 3 & 3 & 3 & 3 & 3 & 4 & 3 & 2 & 3 & 3 & 3 & 3 \\
\hline keller & 3 & 1 & 1 & 1 & 1 & 1 & 1 & 1 & 0 & 0 & 1 & 1 & 1 & 1 \\
\hline MANN & 4 & 1 & 1 & 1 & 2 & 2 & 2 & 3 & 2 & 2 & 1 & 1 & 1 & 1 \\
\hline p_hat & 15 & 5 & 4 & 5 & 7 & 5 & 7 & 4 & 1 & 0 & 8 & 8 & 9 & 11 \\
\hline san & 15 & 7 & 9 & 9 & 8 & 8 & 8 & 13 & 4 & 3 & 6 & 10 & 14 & 15 \\
\hline \multicolumn{2}{|c|}{ DIMACS: 80} & 37 & 38 & 39 & 42 & 40 & 42 & 46 & 22 & 18 & 36 & 48 & 53 & 61 \\
\hline \multicolumn{2}{|c|}{ BHOSLIB: 40} & 0 & 0 & 1 & 0 & 0 & 5 & 3 & 0 & 0 & 0 & 0 & 5 & 5 \\
\hline$(150,0.9)$ & 50 & 43 & 50 & 50 & 50 & 50 & 50 & 50 & 7 & 7 & 50 & 50 & 50 & 50 \\
\hline$(150,0.95)$ & 50 & 46 & 50 & 50 & 49 & 50 & 50 & 50 & 50 & 50 & 0 & 50 & 50 & 50 \\
\hline$(200,0.8)$ & 50 & 38 & 50 & 50 & 49 & 50 & 50 & 50 & 0 & 0 & 50 & 50 & 50 & 50 \\
\hline$(200,0.9)$ & 50 & 44 & 50 & 50 & 49 & 50 & 50 & 50 & 0 & 0 & 0 & 41 & 50 & 50 \\
\hline$(200,0.95)$ & 50 & 44 & 50 & 50 & 49 & 50 & 50 & 50 & 14 & 14 & 0 & 25 & 50 & 50 \\
\hline$(300,0.7)$ & 50 & 30 & 48 & 50 & 50 & 50 & 50 & 50 & 0 & 0 & 50 & 50 & 50 & 50 \\
\hline$(300,0.8)$ & 50 & 14 & 7 & 9 & 47 & 19 & 18 & 41 & 0 & 0 & 50 & 50 & 50 & 50 \\
\hline$(300,0.9)$ & 50 & 8 & 8 & 8 & 2 & 0 & 0 & 35 & 0 & 0 & 0 & 0 & 29 & 50 \\
\hline$(500,0.6)$ & 50 & 0 & 0 & 0 & 0 & 0 & 0 & 0 & 0 & 0 & 50 & 50 & 50 & 50 \\
\hline$(500,0.7)$ & 50 & 0 & 0 & 0 & 0 & 0 & 0 & 0 & 0 & 0 & 49 & 41 & 50 & 50 \\
\hline$(600,0.6)$ & 50 & 0 & 0 & 0 & 0 & 0 & 0 & 0 & 0 & 0 & 50 & 50 & 50 & 50 \\
\hline$(600,0.7)$ & 50 & 0 & 0 & 0 & 0 & 0 & 0 & 0 & 0 & 0 & 0 & 0 & 39 & 50 \\
\hline$(700,0.6)$ & 50 & 0 & 0 & 0 & 0 & 0 & 0 & 0 & 0 & 0 & 50 & 50 & 50 & 50 \\
\hline$(700,0.7)$ & 50 & 0 & 0 & 0 & 1 & 0 & 0 & 0 & 0 & 0 & 0 & 0 & 0 & 50 \\
\hline \multicolumn{2}{|c|}{ RAND: 700} & 267 & 313 & 317 & 346 & 319 & 318 & 376 & 71 & 71 & 399 & 507 & 618 & 700 \\
\hline
\end{tabular}

Experimental results in Table 4 suggest that encoding approaches do affect the performances of MaxSAT solvers to solve an MWC instance. However, the effectiveness of the direct encoding, the split encoding and the iterative encoding for different MaxSAT solvers are not clear. Concretely, the iterative split encoding makes akMaxSat a little faster than other encodings do. MaxHS using the direct encoding dominates MaxHS using other encodings. The iterative split encoding is a better choice for MaxSatz to solve BHOSLIB instances, but the direct encoding is better to solve random graphs. These results also show that MWCLQ significantly outperforms MaxSAT solvers to solve an MWC instance. We observe that although MaxSAT reasoning is powerful in improving the upper bound in a BnB algorithm for MC or MWC, using a MaxSAT solver to solve an MWC instance is not effective. 
Thanks to the extended MaxSAT reasoning, MWCLQ significantly outperforms MWCLQ Mir $_{\text {, }}$

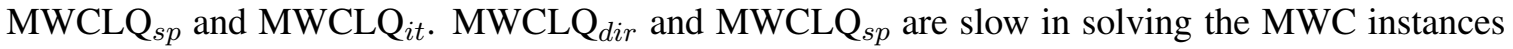
because they cannot capture the graph structure well. Although the iterative split encoding in MWCLQ $_{i t}$ can capture the graph structure, MWCLQ $i t$ does not exploit the power of MaxSAT reasoning efficiently, because the clause splittings in MWCLQ $_{i t}$ are not driven by MaxSAT reasoning.

The advantages of MWCLQ can be described as follows: (1) it is a BnB algorithm for MWC that is able to exploit the graph structure, especially by ordering vertices and by partitioning the graph into independent sets at each search tree node, that a MaxSAT solver does not do; (2) it exploits the power of MaxSAT reasoning in the upper bounding procedure to derive a tight upper bound, that a classical BnB algorithm for MWC does not do. Nevertheless, to make MaxSAT reasoning beneficial, a relevant encoding from MWC into MaxSAT is necessary, as shown in Table 4. The encoding has an obvious impact on the performance of MWCLQ.

\subsection{Effectiveness of the Upper Bound Based on Extended MaxSAT Reasoning}

To study the impact of extended MaxSAT reasoning with soft clause splitting, we implemented two derived versions of MWCLQ, namely, MWCLQ-- and MWCLQ-. MWCLQ-- is identical to MWCLQ except that it only uses the trivial bound based on the independent partition of the graph, which is equal to the sum of the largest weight in each independent set. MWCLQ- is identical to MWCLQ except it only detects the inconsistent subsets of soft clauses, but does not use the $\delta$-Rule and the $(k, \delta)$-Rule to split clauses in the subset.

Table 5 shows runtimes (in seconds) and search tree sizes (in thousands) of MWCLQ--, MWCLQand MWCLQ for solving the DIMACS and BHOSLIB instances, as well as the gain ratio of MWCLQ- and MWCLQ compared with MWCLQ-- in terms of runtime and search tree size computed as MWCLQ--/MWCLQ- and MWCLQ--/MWCLQ, respectively. MaxSAT reasoning without soft clause splitting in MWCLQ- makes MWCLQ- better than MWCLQ-- in terms of search tree size. But the reduction of search tree size is not enough in general to compensate the overhead of MaxSAT reasoning, so that the gain of MWCLQ- compared with MWCLQ-- in terms of runtime is not clear. However, the soft clause splitting using the $\delta$-Rule and the $(k, \delta)$-Rule allows MaxSAT reasoning to prune much more search space, making MWCLQ substantially (from 1.08 to 4.05 times) faster than MWCLQ--. In fact, MWCLQ solves 3 instances more than MWCLQ-- and MWCLQ-, and significantly outperforms them on all instances except san1000. Observe that MWCLQ-- and MWCLQ- solve the same number of instances as Minsatz (58) on the DIMACS benchmark, and solve more instances than Cliquer (52), Dkum (48) and CPLEX (44).

Table 6 shows mean runtimes (in seconds) and mean search tree sizes (in thousands) of different versions of MWCLQ for the random instances, averaged over the solved instances at each point, as well as the gain ratio of MWCLQ and MWCLQ- compared with MWCLQ--. MWCLQ solves all instances. However, neither MWCLQ-- nor MWCLQ- can solve all graphs at the points $(300,0.90)$ and $(700,0.7)$. MaxSAT reasoning allows MWCLQ- to prune more search space than MWCLQ-on all instances, but the overhead makes MWCLQ- slower on instances with more than 300 vertices. However, similarly as in DIMACS and BHOSLIB cases, MWCLQ is from 1.2 to 14.3 times faster than MWCLQ--, because the soft clause splittings using the $\delta$-Rule and the $(k, \delta)$-Rule allow MWCLQ to substantially reduce the search space on all instances. Furthermore, the gain in terms of both runtime and search tree size increases with the density of graph. 
Table 5: Runtimes (in seconds) and search tree sizes (in thousands) of different versions of MWCLQ for solving the DIMACS and BHOSLIB instances, as well as the gain ratio of MWCLQ and MWCLQ- compared with MWCLQ--. The cut-off time is 3600 seconds. '-' means that the solver cannot solve the instance within the cut-off time. Instances solved within 10 seconds by all solvers or not solved by any within the cut-off time are omitted.

\begin{tabular}{|c|c|c|c|c|c|c|c|c|c|c|}
\hline Benchmark & \multicolumn{2}{|c|}{ MWCLQ-- } & \multicolumn{4}{|c|}{ MWCLQ- } & \multicolumn{4}{|c|}{ MWCLQ } \\
\hline Graph & Time & Size & Time & Gain & Size & Gain & Time & Gain & Size & Gain \\
\hline brock400_1 & 209.0 & 63153 & 234.3 & 0.89 & 54505 & 1.16 & 129.3 & 1.62 & 25367 & 2.49 \\
\hline brock400_2 & 203.5 & 58770 & 227.7 & 0.89 & 51244 & 1.15 & 127.7 & 1.59 & 23788 & 2.47 \\
\hline brock400_3 & 173.4 & 50079 & 194.2 & 0.89 & 43616 & 1.15 & 107.2 & 1.62 & 19830 & 2.53 \\
\hline brock400_4 & 131.5 & 37420 & 146.3 & 0.90 & 32853 & 1.14 & 81.81 & 1.61 & 15133 & 2.47 \\
\hline brock800_1 & 1813 & 421259 & 1990 & 0.91 & 396425 & 1.06 & 1427 & 1.27 & 223715 & 1.88 \\
\hline brock800_2 & 2563 & 648486 & 2695 & 0.95 & 605433 & 1.07 & 2002 & 1.28 & 339910 & 1.91 \\
\hline brock800_3 & 1968 & 470594 & 2201 & 0.89 & 442518 & 1.06 & 1545 & 1.27 & 248218 & 1.90 \\
\hline brock800_4 & 2623 & 675790 & 2931 & 0.89 & 633367 & 1.07 & 2039 & 1.29 & 352700 & 1.92 \\
\hline C250.9 & 159.4 & 31542 & 160.5 & 0.99 & 20114 & 1.57 & 39.99 & 3.99 & 4472 & 7.05 \\
\hline DSJC1000.5 & 87.73 & 26154 & 91.87 & 0.95 & 25207 & 1.04 & 81.30 & 1.08 & 17032 & 1.54 \\
\hline gen200_p0.9_44 & 27.83 & 7560 & 23.12 & 1.20 & 3692 & 2.05 & 7.00 & 3.98 & 979 & 7.72 \\
\hline gen200_p0.9_55 & 10.41 & 2530 & 8.70 & 1.20 & 1310 & 1.93 & 2.76 & 3.77 & 344 & 7.34 \\
\hline hamming10-2 & - & - & - & - & - & - & 15.46 & - & 41758 & - \\
\hline p_hat1000-2 & - & - & - & - & - & - & 2501 & - & 176892 & - \\
\hline p_hat500-3 & 3009 & 457787 & 2813 & 1.07 & 238949 & 1.92 & 916.7 & 3.28 & 77115 & 5.94 \\
\hline p_hat700-2 & 113.4 & 17715 & 117.5 & 0.97 & 10571 & 1.68 & 47.88 & 2.37 & 4186 & 4.23 \\
\hline $\operatorname{san} 1000$ & 135.9 & 31173 & 137.2 & 0.99 & 30680 & 1.02 & 183.8 & 0.74 & 21694 & 1.44 \\
\hline san200_0.9_3 & 56.31 & 15955 & 48.47 & 1.16 & 7946 & 2.01 & 16.19 & 3.48 & 2180 & 7.32 \\
\hline san400_0.7_3 & 9.79 & 2936 & 10.53 & 0.93 & 2403 & 1.22 & 6.37 & 1.54 & 1213 & 2.42 \\
\hline san400_0.9_1 & - & - & - & - & - & - & 1257 & - & 63106 & - \\
\hline sanr200_0.9 & 25.90 & 6690 & 20.10 & 1.29 & 3001 & 2.23 & 6.40 & 4.05 & 850 & 7.87 \\
\hline sanr400_0.7 & 35.71 & 12228 & 39.60 & 0.90 & 10797 & 1.13 & 25.57 & 1.40 & 5705 & 2.14 \\
\hline frb30-15-1 & 411.3 & 156311 & 394.8 & 1.04 & 108476 & 1.44 & 244.9 & 1.68 & 27928 & 5.60 \\
\hline frb30-15-2 & 42.28 & 13416 & 39.17 & 1.08 & 9287 & 1.44 & 30.14 & 1.40 & 2597 & 5.17 \\
\hline frb30-15-3 & 171.8 & 67246 & 167.0 & 1.03 & 48401 & 1.39 & 131.7 & 1.30 & 14589 & 4.61 \\
\hline frb30-15-4 & 267.9 & 83139 & 257.3 & 1.04 & 63411 & 1.31 & 181.8 & 1.47 & 16336 & 5.09 \\
\hline frb30-15-5 & 85.27 & 22362 & 82.23 & 1.04 & 17705 & 1.26 & 57.31 & 1.49 & 5575 & 4.01 \\
\hline
\end{tabular}

Table 6: Runtimes (in seconds) and search tree sizes (in thousands) of different versions of MWCLQ for the random instances, averaged over the solved instances at each point, as well as the gain ratio of MWCLQ and MWCLQ- compared with MWCLQ--. The cut-off time is 3600 seconds for a solver to solve one instance.

\begin{tabular}{|c|c|c|c|c|c|c|c|c|c|c|c|c|c|c|}
\hline \multicolumn{2}{|c|}{ Graph } & \multicolumn{3}{|c|}{ MWCLQ-- } & \multicolumn{5}{|c|}{ MWCLQ- } & \multicolumn{5}{|c|}{ MWCLQ } \\
\hline$|V|$ & $D$ & \# & Time & Size & \# & Time & Gain & Size & Gain & \# & Time & Gain & Size & Gain \\
\hline 150 & 0.90 & 50 & 1.85 & 715 & 50 & 1.34 & 1.38 & 255 & 2.81 & 50 & 0.58 & 3.19 & 108 & 6.58 \\
\hline 150 & 0.95 & 50 & 5.23 & 1440 & 50 & 1.28 & 4.09 & 123 & 11.69 & 50 & 0.42 & 12.45 & 46 & 30.69 \\
\hline 200 & 0.80 & 50 & 1.71 & 725 & 50 & 1.89 & 0.90 & 539 & 1.34 & 50 & 0.99 & 1.73 & 245 & 2.96 \\
\hline 200 & 0.90 & 50 & 49.41 & 14030 & 50 & 40.13 & 1.23 & 6109 & 2.30 & 50 & 14.30 & 3.46 & 2031 & 6.91 \\
\hline 200 & 0.95 & 50 & 405.8 & 81772 & 50 & 117.1 & 3.47 & 8583 & 9.53 & 50 & 28.77 & 14.10 & 2454 & 33.32 \\
\hline 300 & 0.70 & 50 & 1.58 & 552 & 50 & 1.80 & 0.88 & 493 & 1.12 & 50 & 1.13 & 1.40 & 263 & 2.10 \\
\hline 300 & 0.80 & 50 & 27.09 & 7655 & 50 & 30.98 & 0.87 & 6265 & 1.22 & 50 & 14.87 & 1.82 & 2611 & 2.93 \\
\hline 300 & 0.90 & 31 & 2261 & 399832 & 33 & 2261 & 1.00 & 245004 & 1.63 & 50 & 845.4 & 2.67 & 85676 & 4.67 \\
\hline 500 & 0.60 & 50 & 7.53 & 2310 & 50 & 8.12 & 0.93 & 2176 & 1.06 & 50 & 6.03 & 1.25 & 1314 & 1.76 \\
\hline 500 & 0.70 & 50 & 133.8 & 34848 & 50 & 149.5 & 0.89 & 31905 & 1.09 & 50 & 93.36 & 1.43 & 16326 & 2.13 \\
\hline 600 & 0.60 & 50 & 42.85 & 13483 & 50 & 46.40 & 0.92 & 12661 & 1.06 & 50 & 34.79 & 1.23 & 7667 & 1.76 \\
\hline 600 & 0.70 & 50 & 1229 & 320607 & 50 & 1390 & 0.88 & 292627 & 1.10 & 50 & 869.9 & 1.41 & 149522 & 2.14 \\
\hline 700 & 0.60 & 50 & 94.10 & 24897 & 50 & 104.8 & 0.90 & 23624 & 1.05 & 50 & 78.24 & 1.20 & 14219 & 1.75 \\
\hline 700 & 0.70 & 31 & 3177 & 682683 & 15 & 3343 & 0.95 & 575774 & 1.19 & 50 & 2434 & 1.31 & 351894 & 1.94 \\
\hline
\end{tabular}




\subsection{Application of MWCLQ for the Winner Determination Problem}

An important application of MWC is to solve the winner determination problem (WDP) in combinatorial auctions. The auctioneer has a set of $m$ items, $M=\{1,2, \ldots, m\}$, to sell, and the buyers submit a set of $n$ bids, $B=\left\{B_{1}, B_{2}, \ldots, B_{n}\right\}$. A bid is a pair $B_{i}=\left(S_{i}, P_{i}\right)$, where $S_{i} \subset M$ is a subset of items and $P_{i} \geq 0$ is a price for all items in $S_{i}$. The WDP problem is to determine the bids as winning or losing so as to maximum the auctioneer's revenue, such that each item can be allocated to at most one bidder. We define an MWC instance $G=(V, E, w)$ for the WDP instance as follows.

- For each bid $B_{i} \in B$, define a vertex $v_{i}$ with weight $w\left(v_{i}\right)=P_{i}$, i.e., $V=\left\{v_{1}, v_{2}, \ldots, v_{n}\right\}$ and $w\left(v_{i}\right)=P_{i}$;

- Add an edge $\left\{v_{i}, v_{j}\right\}$ to $G$ if and only if $B_{i}$ and $B_{j}$ do not share common items, i.e., $E=$ $\left\{\left\{v_{i}, v_{j}\right\} \mid S_{i} \cap S_{j}=\emptyset, 1 \leq i<j \leq n\right\}$.

A maximum weight clique of $G$ corresponds to a feasible subset of bids with a maximum revenue.

In this experiment, we compared MWCLQ with Cliquer, Dkum, MinSatz and CPLEX on realistic WDP instances. We also selected MaxHS using the direct encoding, which is the most effective MaxSAT solver to solve an MWC instance, in this comparison. We used the benchmark provided by Lau and Goh (2002), which has been widely used as benchmark purpose to test WDP algorithms (Guo, Lim, Rodrigues, \& Zhu, 2006; Sghir, Hao, Jaafar, \& Ghédira, 2014; Wu \& Hao, 2015). Instances in this benchmark are generated by incorporating the following factors, i.e., a pricing factor which models a bidder's acceptable price range for each bid, a preference factor which takes into account bidder's preferences among bids, and a fairness factor which measures the fairness in distributing items among bidders. The benchmark contains 500 instances with up to 1500 items and 1500 bids, which can be divided into 5 groups by the item number and the bid number. Each group contains 100 instances labeled as REL-m-n, where $m$ is the number of items and $n$ is the number of bids.

Table 7: Mean runtimes in seconds on WDP instances, obtained by solving 100 graphs of each group. The cut-off time is 3600 seconds. The mean runtime is marked by '-' if no instance is solved in the group within the cut-off time. \# stands for the number of instances solved by the solver.

\begin{tabular}{|c|c|c|c|c|c|c|c|c|c|c|c|c|c|}
\hline \multicolumn{2}{|c|}{ Benchmark } & \multicolumn{2}{c|}{ Cliquer } & \multicolumn{2}{c|}{ DKum } & \multicolumn{2}{c|}{ MinSatz } & \multicolumn{2}{c|}{ MaxHS } & \multicolumn{2}{c|}{ CPLEX } & \multicolumn{2}{c|}{ MWCLQ } \\
Group & $\#$ & Time & $\#$ & Time & $\#$ & Time & $\#$ & Time & $\#$ & Time & $\#$ & Time & $\#$ \\
\hline REL-500-1000 & 100 & 809.9 & 100 & 628.3 & 100 & 552.5 & 100 & - & 0 & - & 0 & $\mathbf{5 5 . 6 1}$ & 100 \\
REL-1000-1000 & 100 & 3.73 & 100 & 4.52 & 100 & 25.39 & 100 & - & 0 & - & 0 & $\mathbf{1 . 4 5}$ & 100 \\
REL-1000-500 & 100 & $\mathbf{0 . 0 3}$ & 100 & $\mathbf{0 . 0 3}$ & 100 & 0.81 & 100 & 1003.7 & 100 & 266.7 & 100 & 0.05 & 100 \\
REL-1000-1500 & 100 & 2.84 & 100 & 3.55 & 100 & 67.90 & 100 & - & 0 & - & 0 & $\mathbf{1 . 5 6}$ & 100 \\
REL-1500-1500 & 100 & 3.38 & 100 & 5.30 & 100 & 78.19 & 100 & - & 0 & - & 0 & $\mathbf{2 . 4 2}$ & 100 \\
\hline
\end{tabular}

Table 7 summarizes the mean runtimes and numbers of instances solved within the cut-off time by group. Results show that MWCLQ outperforms other solvers on all groups except REL-1000500, where Cliquer, Dkum, MinSatz and MWCLQ are comparable. MWCLQ achieves at least $10 X$ speedup for the instances in the hardest group REL-500-1000. MaxHS and CPLEX are not effective in solving these instances. In our experiment, we transformed WDP to MWC first and 
then formulated it with integer programming. Another method is used by Wu and Hao (2015) to formulate WDP directly into integer programming. The method appears to be slightly more effective than the transformation in our experiment, but it does not affect our comparison, because the only instances CPLEX is able to solve within 3600 seconds are also from REL-1000-500 with this transformation.

Table 8 reports the detailed comparative results on the first 10 instances from each group. MWCLQ outperforms other solvers on all instances except the 10 instances from the easiest group (in401, in402, ..., in410), which can be solved by Cliquer, DKum, MinSatz and MWCLQ within less than one second. These results suggest that MWCLQ is more effective in solving MWC instances from WDP than other specific MWC algorithms, MinSatz, MaxHS and CPLEX. Moreover, MWCLQ is even more efficient than some state-of-the-art heuristic algorithms on some relatively hard instances. For example, MWCLQ solves in108 in 101.04 seconds, while the heuristic algorithm (Wu \& Hao, 2015), based on tabu search takes, 113.53 seconds to find the solution with probability 0.73. Ignoring the difference of running environments, MWCLQ is faster than the tabu search algorithm on in108. Note that heuristic algorithms can only give a feasible solution, but cannot guarantee the optimality.

\section{Conclusion}

MaxSAT reasoning has been proved to be very effective for the MC problem, based on a partition of the graph into independent sets. However, MaxSAT reasoning cannot be naturally extended to solve the MWC problem because of the literal weights, as shown by the relatively poor performance of MWCLQ- , MWCLQ $d i r$, MWCLQ $_{s p}$ and MWCLQ $i t$. MWCLQ- exploits MaxSAT reasoning but does not deal with literal weights. The encodings from MWC into MaxSAT used in MWCLQ dir $_{\text {r }}$ and $\mathrm{MWCLQ}_{s p}$ do not capture well the graph structure. Although $\mathrm{MWCLQ}_{i t}$ can exploit the graph structure, it does too many useless splits and has difficulties to take advantage of MaxSAT reasoning efficiently. We thus propose to encode an MWC instance into a literal-weighted MaxSAT instance, in which both soft clauses and literals in soft clauses are weighted. The optimal solution of an LW MaxSAT instance is not an MWC, but an upper bound for the MWC. The interest of the LW encoding is that we can transform an LW MaxSAT instance so that the optimal solution of the new instance is a tighter upper bound for the MWC.

Concretely, at every search tree node of a BnB algorithm for MWC, we partition the current subgraph into independent sets and obtain an LW MaxSAT instance, in which each soft clause corresponds to an independent set. Then we successively transform the LW MaxSAT instance by identifying the Top- $k$ literal failed clause and the Top- $k$ empty clause and by using the $\delta$-Rule and the $(k, \delta)$-Rule. Consequently, we obtain a tight upper bound for MWC to prune the search space. This approach is implemented in MWCLQ, which is substantially better than MWCLQ-,

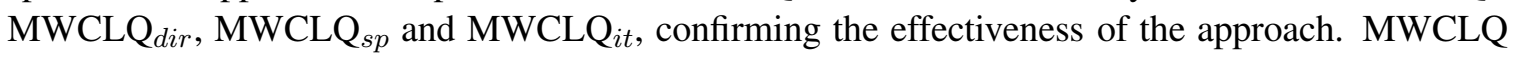
is also favorably compared with the state-of-the-art MWC solvers as Cliquer, DKum, MinSatz and CPLEX, as well as several state-of-the-art MaxSAT solvers using different encodings, on standard benchmarks and instances from realistic applications.

In the future, we plan to study the impact of vertex ordering and unit clause ordering in MWCLQ. It is also interesting to use MaxSAT reasoning to solve other combinatorial optimization problems, especially the weighted version, using a dedicated encoding. 
Table 8: Runtimes in seconds on the first 10 instances of each group in the benchmark in Table 7. The cut-off time is 3600 seconds. $|V|$ stands for the number of vertices, $D$ for the density of the graph after transforming WDP into MWC and $\omega_{v}$ for the optimal solution of MWC. When a solver cannot solve an instance within 3600 seconds, its runtime is marked by '-'

\begin{tabular}{|c|c|c|c|c|c|c|c|c|c|}
\hline Graph & $|V|$ & $\mathrm{D}$ & $\omega_{v}$ & Cliquer & DKum & MinSatz & MaxHS & CPLEX & MWCLQ \\
\hline in101 & 1000 & 0.31 & 72724.617 & 819.6 & 616.1 & 610.8 & - & - & 53.87 \\
\hline in 102 & 1000 & 0.30 & 72518.222 & 414.9 & 289.0 & 308.8 & - & - & 34.40 \\
\hline in 103 & 1000 & 0.31 & 72129.500 & 551.4 & 455.5 & 479.6 & - & - & 44.94 \\
\hline in104 & 1000 & 0.30 & 72709.646 & 330.8 & 218.1 & 353.5 & - & - & 37.00 \\
\hline in105 & 1000 & 0.30 & 75646.127 & 840.3 & 547.3 & 367.7 & - & - & 37.33 \\
\hline in106 & 1000 & 0.30 & 71258.613 & 272.7 & 346.4 & 270.7 & - & - & 23.56 \\
\hline in107 & 1000 & 0.30 & 69713.403 & 595.9 & 460.1 & 736.9 & - & - & 68.07 \\
\hline in108 & 1000 & 0.31 & 75813.205 & 1321 & 1121 & 924.9 & - & - & 101.04 \\
\hline in109 & 1000 & 0.30 & 69475.895 & 247.3 & 238.8 & 305.0 & - & - & 29.10 \\
\hline in 110 & 1000 & 0.30 & 68295.289 & 308.5 & 307.7 & 411.7 & - & - & 43.16 \\
\hline in201 & 1000 & 0.15 & 81557.742 & 1.80 & 2.47 & 22.09 & - & - & 1.01 \\
\hline in 202 & 1000 & 0.16 & 90708.127 & 3.73 & 4.98 & 25.90 & - & - & 1.37 \\
\hline in 203 & 1000 & 0.16 & 86239.214 & 3.63 & 6.65 & 26.66 & - & - & 1.57 \\
\hline in204 & 1000 & 0.17 & 87075.428 & 6.13 & 7.20 & 30.47 & - & - & 2.01 \\
\hline in205 & 1000 & 0.16 & 86515.951 & 3.11 & 4.85 & 27.42 & - & - & 1.64 \\
\hline in206 & 1000 & 0.16 & 91518.964 & 2.37 & 3.37 & 22.85 & - & - & 1.11 \\
\hline in207 & 1000 & 0.17 & 93129.248 & 3.95 & 5.69 & 28.18 & - & - & 1.69 \\
\hline in208 & 1000 & 0.16 & 94904.679 & 4.07 & 5.18 & 22.09 & - & - & 1.05 \\
\hline in209 & 1000 & 0.16 & 87268.965 & 2.13 & 3.38 & 26.66 & - & - & 1.54 \\
\hline in210 & 1000 & 0.16 & 89962.396 & 3.54 & 4.78 & 22.09 & - & - & 1.21 \\
\hline in401 & 500 & 0.15 & 77417.482 & 0.02 & 0.03 & 0.69 & 923.01 & 181.70 & 0.04 \\
\hline in 402 & 500 & 0.15 & 76273.336 & 0.02 & 0.02 & 0.72 & 1107.2 & 198.12 & 0.04 \\
\hline in 403 & 500 & 0.16 & 74843.957 & 0.03 & 0.04 & 0.76 & 891.23 & 229.0 & 0.04 \\
\hline in404 & 500 & 0.17 & 78761.690 & 0.05 & 0.08 & 0.89 & 1082.9 & 208.97 & 0.06 \\
\hline in 405 & 500 & 0.17 & 75915.900 & 0.05 & 0.08 & 0.88 & 1300.6 & 224.98 & 0.06 \\
\hline in 406 & 500 & 0.14 & 72863.324 & 0.02 & 0.02 & 0.75 & 992.34 & 175.15 & 0.05 \\
\hline in 407 & 500 & 0.17 & 76365.717 & 0.05 & 0.07 & 0.79 & 1569.1 & 364.58 & 0.06 \\
\hline in 408 & 500 & 0.16 & 77018.833 & 0.04 & 0.04 & 0.82 & 1187.2 & 199.39 & 0.06 \\
\hline in 409 & 500 & 0.14 & 73188.619 & 0.01 & 0.02 & 0.71 & 846.56 & 217.31 & 0.04 \\
\hline in 410 & 500 & 0.17 & 73791.658 & 0.04 & 0.06 & 0.85 & 1132.1 & 243.42 & 0.06 \\
\hline in501 & 1500 & 0.09 & 88656.958 & 4.64 & 5.68 & 81.25 & - & - & 2.30 \\
\hline in502 & 1500 & 0.08 & 86236.911 & 1.69 & 2.30 & 65.16 & - & - & 1.45 \\
\hline in503 & 1500 & 0.08 & 87812.377 & 3.44 & 4.42 & 72.06 & - & - & 1.72 \\
\hline in504 & 1500 & 0.08 & 85600.001 & 2.37 & 3.80 & 61.32 & - & - & 1.56 \\
\hline in505 & 1500 & 0.08 & 84860.165 & 2.16 & 2.82 & 62.09 & - & - & 1.56 \\
\hline in506 & 1500 & 0.08 & 84623.414 & 1.41 & 2.32 & 65.92 & - & - & 1.34 \\
\hline in507 & 1500 & 0.08 & 90288.472 & 2.82 & 3.73 & 68.99 & - & - & 1.32 \\
\hline in508 & 1500 & 0.07 & 86853.500 & 1.24 & 1.60 & 58.26 & - & - & 1.11 \\
\hline in509 & 1500 & 0.08 & 88316.087 & 3.59 & 4.37 & 64.39 & - & - & 1.80 \\
\hline in510 & 1500 & 0.08 & 89014.137 & 1.29 & 2.19 & 64.39 & - & - & 1.15 \\
\hline in601 & 1500 & 0.09 & 108800.445 & 4.52 & 5.51 & 76.04 & - & - & 2.39 \\
\hline in602 & 1500 & 0.09 & 105611.476 & 2.01 & 2.98 & 70.61 & - & - & 1.75 \\
\hline in603 & 1500 & 0.09 & 105121.021 & 1.84 & 2.56 & 65.96 & - & - & 1.35 \\
\hline in604 & 1500 & 0.10 & 107733.805 & 3.86 & 5.71 & 84.58 & - & - & 3.09 \\
\hline in605 & 1500 & 0.10 & 109840.984 & 3.72 & 5.75 & 75.27 & - & - & 2.22 \\
\hline in606 & 1500 & 0.10 & 107113.067 & 2.72 & 4.37 & 77.60 & - & - & 2.13 \\
\hline in607 & 1500 & 0.10 & 113180.284 & 4.20 & 6.65 & 78.37 & - & - & 2.38 \\
\hline in608 & 1500 & 0.10 & 105266.107 & 2.21 & 3.72 & 77.60 & - & - & 2.17 \\
\hline in609 & 1500 & 0.10 & 109472.332 & 2.77 & 3.78 & 69.84 & - & - & 1.86 \\
\hline in610 & 1500 & 0.11 & 113716.965 & 6.14 & 10.03 & 94.67 & - & - & 3.70 \\
\hline
\end{tabular}




\section{Acknowledgments}

We would like to thank anonymous reviewers for their helpful comments and suggestions. We also thank Jichang Zhao, Qiao Kan, Xu Feng and Shaowei Cai for their proofreads and suggestions. Part of this work was done while the first author was a joint Ph.D. student at Université de Picardie Jules Verne. This research was partly supported by NSFC (Grant No. 61421003), the fund of the State Key Lab of Software Development Environment (Grant No. SKLSDE-2015ZX-05), the Chinese State Key Laboratory of Software Development Environment Open Fund (Grant No. SKLSDE2012KF-07), and the MeCS platform of Université de Picardie Jules Verne.

\section{References}

Abramé, A., \& Habet, D. (2014). Efficient application of max-sat resolution on inconsistent subsets. In Proc. of CP-2014, pp. 92-107. Springer.

Abramé, A., \& Habet, D. (2015). On the resiliency of unit propagation to max-resolution. In Proc. of AAAI-2015, pp. 268-274. AAAI Press.

Ansótegui, C., Bonet, M. L., \& Levy, J. (2009). Solving (weighted) partial maxsat through satisfiability testing. In Theory and Applications of Satisfiability Testing-SAT 2009, pp. 427-440.

Ansótegui, C., Bonet, M. L., \& Levy, J. (2013). Sat-based maxsat algorithms. Artificial Intelligence, 196, 77-105.

Ansótegui, C., \& Gabas, J. (2013). Solving (weighted) partial maxsat with ILP. In Proc. of CPAIOR2013, pp. 403-409.

Benlic, U., \& Hao, J. K. (2013). Breakout local search for maximum clique problems. Computers \& Operations Research, 40, 192-206.

Bonet, M. L., Levy, J., \& Manyà, F. (2006). A complete calculus for max-sat. In Theory and Applications of Satisfiability Testing-SAT 2006, pp. 240-251. Springer.

Cai, S., Su, K., Luo, C., \& Sattar, A. (2013). NuMVC: An efficient local search algorithm for minimum vertex cover. Journal of Artificial Intelligence Research, 46, 687-716.

Cai, S., Su, K., \& Sattar, A. (2011). Local search with edge weighting and configuration checking heuristics for minimum vertex cover. Artificial Intelligence, 175(9), 1672-1696.

Chen, J. (2010). A new SAT encoding of the at-most-one constraint. In International Workshop on Modelling and Reformulating Constraint Satisfaction Problems.

Davies, J., \& Bacchus, F. (2013a). Exploiting the power of mip solvers in maxsat. In Theory and Applications of Satisfiability Testing-SAT 2013, pp. 166-181. Springer.

Davies, J., \& Bacchus, F. (2013b). Postponing optimization to speed up MAXSAT solving. In Proc. of CP-2013, pp. 247-262. Springer.

Downey, R. G., \& Fellows, M. R. (1995). Fixed-parameter tractability and completeness I: Basic results. SIAM Journal on Computing, 24(4), 873-921.

Fahle, T. (2002). Simple and fast: Improving a branch-and-bound algorithm for maximum clique. In Proc. of ESA-2002, pp. 485-498.

Fang, Z., Chu, Y., Qiao, K., Feng, X., \& Xu, K. (2014a). Combining edge weight and vertex weight for minimum vertex cover problem. In Frontiers in Algorithmics, pp. 71-81. Springer. 
Fang, Z., Li, C. M., Qiao, K., Feng, X., \& Xu, K. (2014b). Solving maximum weight clique using maximum satisfiability reasoning. In Proc. of ECAI-2014, Vol. 263, pp. 303 - 308.

Feige, U. (2004). Approximating maximum clique by removing subgraphs. SIAM Journal on Discrete Mathematics, 18(2), 219-225.

Freeman, J. W. (1995). Improvements to propositional satisfiability search algorithms. Ph.D. thesis.

Guo, Y., Lim, A., Rodrigues, B., \& Zhu, Y. (2006). Heuristics for a bidding problem. Computers \& operations research, 33(8), 2179-2188.

Ignatiev, A., Morgado, A., \& Marques-Silva, J. (2014). On reducing maximum independent set to minimum satisfiability. In Theory and Applications of Satisfiability Testing-SAT 2014, pp. 103-120. Springer.

Karp, R. M. (1972). Reducibility among combinatorial problems. In Complexity of Computer Computations, pp. pp 85-103. Springer.

Kibanov, M., Atzmueller, M., Scholz, C., \& Stumme, G. (2014). Temporal evolution of contacts and communities in networks of face-to-face human interactions. Science China Information Sciences, 57(3), 1-17.

Konc, J., \& Janezic, D. (2007). An improved branch and bound algorithm for the maximum clique problem. Communications in Mathematical and in Computer Chemistry, 58, 569-590.

Kügel, A. (2010). Improved exact solver for the weighted Max-SAT problem. In Workshop Pragmatics of SAT, Vol. 436.

Kügel, A. (2012). Natural Max-SAT encoding of Min-SAT. In Learning and Intelligent Optimization, pp. 431-436. Springer.

Kumlander, D. (2004). A new exact algorithm for the maximum-weight clique problem based on a heuristic vertex-coloring and a backtrack search. In Proc. of MOC-2004, pp. 202-208.

Kumlander, D. (2008a) http://www.kumlander.eu/graph/index.html.

Kumlander, D. (2008b). On importance of a special sorting in the maximum-weight clique algorithm based on colour classes. In Modelling, computation and optimization in information systems and management sciences, pp. 165-174. Springer.

Larrosa, J., Heras, F., \& de Givry, S. (2008). A logical approach to efficient max-sat solving. Artificial Intelligence, 172(2), 204-233.

Lau, H. C., \& Goh, Y. G. (2002). An intelligent brokering system to support multi-agent web-based 4 th-party logistics. In Proc. of ICTAI-2002, pp. 154-161. IEEE.

Li, C. M., \& Anbulagan, A. (1997). Heuristics based on unit propagation for satisfiability problems. In Proc. of the IJCAI-1997, pp. 366-371. Morgan Kaufmann Publishers Inc.

Li, C. M., Fang, Z., \& Xu, K. (2013). Combining MaxSAT reasoning and incremental upper bound for the maximum clique problem. In Proc. of ICTAI-2013, pp. 939-946. IEEE.

Li, C. M., Manyà, F., \& Planes, J. (2005). Exploiting unit propagation to compute lower bounds in branch and bound max-sat solvers. In Proc. of CP-2005, Vol. 3709, pp. 403-414. Springer.

Li, C. M., Manyà, F., \& Planes, J. (2007). New inference rules for Max-SAT. Journal of Artificial Intelligence Research, 30, 321-359. 
Li, C. M., \& Manya, F. (2009). Maxsat, hard and soft constraints.. Handbook of satisfiability, 185, 613-631.

Li, C. M., Manya, F., \& Planes, J. (2006). Detecting disjoint inconsistent subformulas for computing lower bounds for max-sat. In Proc. of AAAI-2006, Vol. 6, pp. 86-91.

Li, C. M., \& Quan, Z. (2010a). Combining graph structure exploitation and propositional reasoning for the maximum clique problem. In Proc. of ICTAI-2010, Vol. 1, pp. 344-351. IEEE.

Li, C. M., \& Quan, Z. (2010b). An efficient branch-and-bound algorithm based on MaxSAT for the maximum clique problem. In Proc. of AAAI-2010, pp. 128-133.

Li, C. M., Zhu, Z., Manyà, F., \& Simon, L. (2012). Optimizing with minimum satisfiability. Artificial Intelligence, 190, 32-44.

Liu, T., Lin, X., Wang, C., Su, K., \& Xu, K. (2011). Large hinge width on sparse random hypergraphs. In Proc. of IJCAI-2011, Vol. 2011, pp. 611-616.

Ma, T., \& Latecki, L. J. (2012). Maximum weight cliques with mutex constraints for video object segmentation. In Proc. of CVPR-2012, pp. 670-677. IEEE.

Martins, R., Joshi, S., Manquinho, V., \& Lynce, I. (2014). Incremental cardinality constraints for maxsat. In Proc. of CP-2014, pp. 531-548. Springer.

Mascia, F., Cilia, E., Brunato, M., \& Passerini, A. (2010). Predicting structural and functional sites in proteins by searching for maximum-weight cliques. In Proc. of AAAI-2010, pp. 1274-1279. AAAI.

Morgado, A., Dodaro, C., \& Marques-Silva, J. (2014). Core-guided maxsat with soft cardinality constraints. In Proc. of CP-2014, pp. 564-573. Springer.

Morgado, A., Heras, F., Liffiton, M., Planes, J., \& Marques-Silva, J. (2013). Iterative and coreguided maxsat solving: A survey and assessment. Constraints, 18(4), 478-534.

Östergård, P. (2001). A new algorithm for the maximum-weight clique problem. Nordic Journal of Computing, 8, 424-436.

Östergård, P. (2002). A fast algorithm for the maximum clique problem. Discrete Applied Mathematics, 120, 197-207.

Östergård, P. (2010). Cliquer source code. http://users.tkk. fi/pat/cliquer.html.

Pullan, W. (2008). Approximating the maximum vertex/edge weighted clique using local search. Journal of Heuristics, 19, 117-134.

Pullan, W., \& Hoos, H. H. (2006). Dynamic local search for the maximum clique problem. Journal of Artificial Intelligence Research, 25, 159-185.

Régin, J. C. (2003). Solving the maximum clique problem with constraint programming. In Proc. of CPAIOR-2003, pp. 634-648.

Sghir, I., Hao, J.-K., Jaafar, I. B., \& Ghédira, K. (2014). A recombination-based tabu search algorithm for the winner determination problem. In Artificial Evolution, pp. 157-167. Springer.

Shimizu, S., Yamaguchi, K., Saitoh, T., \& Masuda, S. (2012). Some improvements on Kumlander's maximum weight clique extraction algorithm. In Proc. of the International Conference on Electrical, Computer, Electronics and Communication Engineering, pp. 307-311. 
Shimizu, S., Yamaguchi, K., Saitoh, T., \& Masuda, S. (2013). Optimal table method for finding the maximum weight clique. In Proc. of the 13th International Conference on Applied Computer Science, No. 12. WSEAS.

ThanhVu, H. N., \& Thang, B. (2014). DIMACS benchmark. https : / / turing • cs . hbg • psu . edu/txn131/clique.html.

Tomita, E., \& Kameda, T. (2007). An efficient branch-and-bound algorithm for finding a maximum clique with computational experiments. Journal of Global Optimization, 37, 95-111.

Tomita, E., \& Seki, T. (2003). An efficient branch-and-bound algorithm for finding a maximum clique. In Proc. Discrete Mathematics and Theoretical Computer Science, Vol. 2731, pp. 278-289.

Wu, Q., Hao, J. K., \& Glover, F. (2012). Multi-neighborhood tabu search for the maximum weight clique problem. Annals of Operations Research, 196, 611-634.

Wu, Q., \& Hao, J.-K. (2015). Solving the winner determination problem via a weighted maximum clique heuristic. Expert Systems with Applications, 42(1), 355-365.

Xu, K., Boussemart, F., Hemery, F., \& Lecoutre, C. (2007). Random constraint satisfaction: Easy generation of hard (satisfiable) instances. Artificial Intelligence, 171, 514-534.

$\mathrm{Xu}, \mathrm{K} .$, \& Li, W. (2000). Exact phase transitions in random constraint satisfaction problems. Journal of Artificial Intelligence Research, 12, 93-103.

Xu, K. (2004). BHOSLIB: Benchmarks with hidden optimum solutions for graph problems. http: //www.nlsde.buaa.edu.cn/ kexu/benchmarks/graph-benchmarks.htm.

$\mathrm{Xu}, \mathrm{K} .$, \& Li, W. (2006). Many hard examples in exact phase transitions. Theoretical Computer Science, 355(3), 291-302.

Yamaguchi, K., \& Masuda, S. (2008). A new exact algorithm for the maximum weight clique problem. In Proc. of ITC-CSCC-2008, pp. 317-320.

Zhang, D., Javed, O., \& Shah, M. (2014a). Video object co-segmentation by regulated maximum weight cliques. In Proc. of ECCV-2014, pp. 551-566. Springer.

Zhang, W., Nie, L., Jiang, H., Chen, Z., \& Liu, J. (2014b). Developer social networks in software engineering: construction, analysis, and applications. Science China Information Sciences, 57(12), 1-23.

Zhian, H., Sabaei, M., Javan, N. T., \& Tavallaie, O. (2013). Increasing coding opportunities using maximum-weight clique. In Proc. of Computer Science and Electronic Engineering Conference-2013, pp. 168-173. IEEE.

Zhu, Z., Li, C. M., Manyà, F., \& Argelich, J. (2012). A new encoding from MinSAT into MaxSAT. In Proc. of CP-2012, pp. 455-463. Springer.

Zuckerman, D. (2006). Linear degree extractors and the inapproximability of max clique and chromatic number. In Proceedings of the 38th annual ACM symposium on Theory of computing, pp. 681-690. ACM. 